\title{
Le modalità logico-argomentative nei testi scolastici di geometria della scuola elementare e media in lingua italiana
}

\section{The logical-argumentative modalities in Italian language primary and lower secondary school geometry textbooks}

\author{
Silvia Sbaragli ${ }^{\bullet}$ Michele Canducci॰॰ e Silvia Demartini ${ }^{\bullet}$ \\ - Dipartimento formazione e apprendimento, SUPSI - Locarno, Svizzera \\ - Facoltà di comunicazione, cultura e società, USI - Lugano, Svizzera \\ 凶silvia.sbaragli@supsi.ch, michele.canducci@supsi.ch, silvia.demartini@supsi.ch
}

Sunto / In quest'articolo si intende portare l'attenzione sulle modalità logico-argomentative presenti nei testi scolastici di matematica, focalizzandosi sulla parte di geometria e, nello specifico, sul tema poligoni, considerando la ricorsività dell'argomento in continuità fra gli ordini scolastici (dalla II elementare alla III media). L'indagine è strettamente interdisciplinare fra matematica e linguistica, con particolare attenzione alla didattica, e si inserisce nei lavori di un più vasto progetto di ricerca in corso. Dopo alcuni paragrafi iniziali dedicati a illustrare il corpus di libri e i criteri di analisi del testo adottati, si passerà a una parte di inquadramento storico-disciplinare del tema, per addentrarsi poi nella descrizione delle diverse modalità logico-argomentative (legate al far "fare", al far "immaginare" e al far "astrarre"); di queste saranno anche offerti dati quantitativi relativi alla distribuzione nel corpus. Alcune possibili implicazioni didattiche emerse dalle analisi saranno accennate nelle conclusioni.

Parole chiave: argomentazione; libri di testo scolastici di matematica; discorso matematico; poligoni; linguistica testuale.
Abstract / This article analyses the logical-argumentative strategies contained in mathematics schoolbooks, focusing on geometry and, specifically, on polygons, considering the recursiveness of the topic in continuity between school orders. The investigation is strictly interdisciplinary between mathematics and linguistics, with particular attention for didactic, and is part of a larger ongoing research project. After some initial paragraphs devoted to describing the corpus and the text analysis criteria, we will move on to a historical-disciplinary framework of the topic, and then to the definition of the different logical-argumentative modes (linked to "doing", "imagining" and "abstracting"); quantitative data about the distribution in the corpus will also be offered. Some possible teaching implications emerging from the analyses will be mentioned in the conclusions.

Keywords: argumentation; mathematics textbooks; mathematical discourse; polygons; text linguistics. 


\section{L'oggetto di analisi e il corpus di testi in esame}

II tema trattato in questo contributo si inserisce all'interno del progetto Italmatica. Comprendere la matematica a scuola, fra lingua comune e linguaggio specialistico (progetto 176339 del Fondo nazionale svizzero per la ricerca scientifica). Grazie a un gruppo eterogeneo di ricercatori in didattica della matematica, linguistica e computer science, il progetto ha l'obiettivo di individuare, raccogliere e analizzare, dal punto di vista linguistico e matematico, un corpus di libri di testo scolastici di matematica in lingua italiana della scuola elementare e media, al fine di delinearne le caratteristiche e i possibili ostacoli per la comprensione degli alunni.

L'argomento matematico su cui si è concentrata l'attenzione è interno all'ambito geometrico e riguarda i poligoni, in un'ottica di continuità dalla scuola elementare alla scuola media. L'argomento dei poligoni, infatti, permea tutta la scuola dell'obbligo, in accordo con l'idea di percorso a spirale per la costruzione di competenze matematiche, in cui alcuni degli argomenti affrontati dagli allievi nei primi anni di scolarità vengono consolidati e approfonditi in diverse occasioni negli anni successivi. Questa scelta ci ha permesso di raccogliere libri di testo riferiti a sette anni di scolarità, dalla seconda elementare alla terza media, arrivando a comporre una panoramica multiforme e sfaccettata, all'interno della quale convivono elementi di eterogeneità e di unitarietà.

Dal punto di vista classificatorio, il libro di testo sfugge a categorizzazioni nette. A livello di funzione prevalente, è tipicamente considerato un testo di tipo informativo (o espositivo), o, ancora meglio, «espositivo-esplicativo» (A. Ferrari, 2019, p. 78), e presenta, com'è noto, tratti ricorrenti: veri e propri stilemi e modalità espressive sedimentate nel tempo. È però altrettanto noto che non si tratta di un genere omogeneo al suo interno, ma ibrido: ciò è più che mai evidente nei testi di matematica, che alternano parti caratterizzate da uno stile vicino alla saggistica disciplinare classica a parti più vicine alla lingua comune, dedicate, per esempio, a sollecitare il giovane lettore evocando legami con l'esperienza diretta. Anche a causa delle sue stesse caratteristiche, il testo scolastico, si trova a essere sì un oggetto di riferimento in didattica, ma non sempre funzionale e per questo non pienamente sfruttato dai docenti, o, almeno, non in tutte le sue parti (sull'uso del libro di testo di matematica da parte dei docenti italiani e ticinesi, si vedano Canducci et al., 2020; Sbaragli et al., 2020). Analizzarne più a fondo le modalità comunicative ${ }^{1}$ può quindi essere un primo passo per capirne meglio limiti e punti di forza, anche nella prospettiva di rendere più consapevoli i docenti.

In questo contributo, nel par. 2 presenteremo il modello di analisi testuale dei libri scolastici di matematica da noi adottato, cosa che ci permetterà di focalizzare l'attenzione sul tipo di Movimento Testuale oggetto di analisi, da noi chiamato logico-argomentativo. Nel par. 3 verrà inquadrato il tema dell'argomentazione in chiave interdisciplinare, mettendo in evidenza alcuni dei tanti risvolti che questo ambito d'indagine ha avuto nella storia del pensiero e della cultura. Ciò nella consapevolezza che l'argomentazione è un tema di vastissima portata, su cui sin dall'antichità classica si sono concentrate le riflessioni di filosofi e matematici (le cui figure spesso coincidevano), e con cui, nei secoli e per vari fini, si sono confrontati studiosi di vari ambiti, con crescente attenzione da parte delle diverse discipline. Questa longevità e certe peculiarità della tradizione occidentale (anche rispetto alla prassi scolastica) conferiscono al tema argomentazione notevole importanza nella ricerca in didattica, soprattutto negli ultimi decenni, se si considera che la competenza argomentativa è considerata oggi

1. Data la natura interdisciplinare del progetto - che indaga la didattica della matematica attraverso l'esame degli aspetti linguistici in essa coinvolti -, il gruppo di ricerca ha potuto effettuare analisi sui testi a più livelli, di tipo sia quantitativo sia qualitativo. Le analisi dei libri di testo si sono per ora concentrate sulle seguenti dimensioni: aspetti strutturali di architettura testuale dei testi scolastici (Demartini, Sbaragli \& Ferrari, 2020); aspetti lessicali e morfosintattici dei testi scolastici (Canducci at al., 2019a, 2019b; Canducci, Demartini \& Sbaragli, in stampa; Demartini, Fornara \& Sbaragli, 2020; Demartini \& Sbaragli, 2019); aspetti legati al rapporto multimodale fra testo e figure nei manuali (Canducci, 2019; Canducci, Rocci \& Sbaragli, in stampa). 
un traguardo condiviso esplicito dalle varie discipline (e segnatamente dalla matematica) nei vari piani di studio/indicazioni nazionali, e nelle prove standardizzate nazionali e internazionali. Dopo questo quadro storico e interdisciplinare, nel par. 4 verrà presentata I'analisi del Movimento Testuale logico-argomentativo peculiare dei testi matematici, esplicitando le sue diverse possibilità di realizzazione osservate nei testi, attraverso l'enucleazione di esempi che consentono di capirne meglio la natura sfaccettata. Verranno in seguito presentati i risultati dell'analisi condotta su tutto il corpus di libri di testo italiani e svizzeri, andando a evidenziare l'evoluzione delle diverse categorie di Movimenti logico-argomentativi lungo i sette anni di scolarità considerati per poi giungere alle conclusioni.

\section{Analisi testuale dei libri scolastici di matematica}

L'analisi dei testi scolastici di matematica si basa su un adattamento del modello di analisi della testualità elaborato dal gruppo di ricerca basilese, descritto in A. Ferrari $(2014,2019)$. Tale modello considera il testo come composto da una sequenza di unità semantiche, organizzate gerarchicamente su tre livelli (tralasciando, qui, le partizioni di ordine superiore, come i capitoli e i paragrafi): «i Movimenti Testuali, a loro volta segmentati in Enunciati, i quali sono composti da Unità Informative» (A. Ferrari, 2019, p. 34). II Movimento Testuale consiste in una sequenza di Enunciati nella quale è riconoscibile un'unitarietà dal punto di vista tematico-referenziale o dal punto di vista logico. Esso è il risultato di un macro-atto di composizione testuale, e la sua segnalazione linguistica può variare a seconda del genere di testo considerato; nel caso di un testo scolastico di matematica, i confini di un Movimento Testuale possono essere segnalati da una varietà di espedienti grafici il cui fine è quello di orientare il lettore nell'individuazione di blocchi di testo definiti (Demartini, Sbaragli \& Ferrari, 2020).

A livello qualitativo, dall'analisi del corpus si è potuto notare che i Movimenti Testuali presenti in un libro di testo di matematica fanno principalmente riferimento a due intenti comunicativi: far sapere qualcosa al lettore e far fare qualcosa al lettore. Nel primo caso si parlerà di Movimento espositivoesplicativo, nel secondo di Movimento direttivo. A sua volta, il Movimento espositivo-esplicativo è stato differenziato in tre tipi, corrispondenti a differenti realizzazioni dell'intento comunicativo: il Movimento espositivo-esplicativo di tipo dichiarativo, che tipicamente propone una o più asserzioni; il Movimento espositivo-esplicativo di tipo narrativo-descrittivo, nel quale vengono approfonditi concetti attraverso descrizioni di giochi, excursus storici, inserti etimologici; infine il Movimento espositivo-esplicativo di tipo logico-argomentativo, cioè

«un macro-atto che non offre semplici dichiarazioni, ma che accompagna il ragionamento del lettore nella costruzione del sapere o che comunque cerca di favorire l'interiorizzazione di esso attraverso prove, sperimentazioni (concrete o simulate) e confronti finalizzati a comprendere e a supportare un'asserzione».

(Demartini, Sbaragli \& Ferrari, 2020, p. 168)

Come si è detto, in questo contributo ci concentreremo solo su quest'ultimo tipo di Movimento Testuale, con l'intento di delinearne le caratteristiche, le modalità di realizzazione a livello disciplinare e linguistico, la presenza nei manuali del corpus a livello quantitativo, la sua evoluzione nel corso degli anni di scolarità. Ci concentreremo su di esso consapevoli di quanto la categoria sia scivolosa, soprattutto per i contorni sfumati che spesso assume nei libri di testo, nei quali inevitabilmente possiede simultaneamente un fine argomentativo ma anche esplicativo, ma altrettanto consapevoli di quanto essa sia fondamentale per osservare come i lettori vengano accompagnati nella costruzione concet- 
tuale. Non a caso, come si è detto, tali Movimenti appartengono alla famiglia dei macro-atti espositivoesplicativi, tipici del discorso scientifico disciplinare offerto dai testi per la scuola. Per procedere, è prima utile affrontare in una prospettiva più ampia, seppur brevemente, il tema dell'argomentazione da alcuni punti di vista utili allo scopo dell'articolo.

\section{L'argomentazione in chiave interdisciplinare e didattica}

\subsection{Verso la teoria dell'argomentazione: breve introduzione storico-culturale}

La storia della cultura occidentale è contraddistinta da alcuni elementi forti e caratterizzanti, che ne determinano a lungo termine i modi di vedere le cose e anche di impostare la formazione delle giovani generazioni. Tra questi elementi vi sono la retorica e la dialettica (inizialmente viste come discipline distinte, anche se complementari), le cui riflessioni rientrano attualmente all'interno degli studi che orbitano attorno alla teoria dell'argomentazione. I primi studi di retorica hanno origine nella Magna Grecia del V secolo a.C. grazie alla nascita di un vasto movimento filosofico che va sotto il nome di sofistica. La retorica, intesa come arte di saper parlare per convincere gli altri delle proprie ragioni (ricorrendo non per forza ad argomenti veri, ma anche solo verosimili), è uno dei capisaldi di tale movimento: mediante la carica persuasiva della parola, infatti, i sofisti hanno insegnato la morale, le leggi, i sistemi politici. A differenza dei filosofi greci precedenti, non si interessano alla cosmologia e alla ricerca dell'arché originario, ma si concentrano, piuttosto, sulla vita umana, diventando così i primi filosofi morali.

Ma sarà solo un secolo più tardi, attraverso il lavoro di sistemazione compiuto da Aristotele nell'Organon e nella Retorica, che verrà tracciato un solco imprescindibile per tutti gli sviluppi teorici successivi. Nel lavoro di Aristotele si possono distinguere diverse aree di studio: I'analitica, nella quale si mettono in luce i meccanismi della deduzione che parte da premesse vere per giungere a conclusioni logicamente fondate e necessariamente vere, e che andranno a costituire la base su cui si svilupperà tutto il pensiero logico-deduttivo matematico (D'Amore \& Sbaragli, 2017); la dialettica, parallela all'analitica, ma che a differenza di questa non si occupa del necessariamente vero bensì del verosimile, ossia di opinioni, di tesi cui si aderisce con intensità variabile, ed è concepita dallo stesso Aristotele "come I'arte di ragionare partendo da opinioni generalmente accettate» (Perelman \& Olbrechts-Tyteca, 1958/2013, p. 7); infine la retorica, intesa da Aristotele come complementare alla dialettica, ha come oggetto il ragionamento persuasivo nei confronti di un uditorio, e insegna come si deve dire qualcosa (secondo quali schemi, seguendo quali criteri e con quali cautele) allo scopo di rendere efficace ciò che viene detto-fatto, producendo determinati effetti sull'uditore: convincerlo circa la credibilità di un'opinione, indurlo a compiere o ad astenersi dal compiere una data azione, portarlo a modificare certi suoi atteggiamenti, sentimenti ecc. (Cattani, 1994).

La tradizione retorica ebbe importanti sviluppi per tutta I'antichità classica (Mortara Garavelli, 2003), laddove il discorso persuasivo (quindi un certo tipo di argomentazione) rappresentava il cuore del dibattito pubblico e della vita civile. ${ }^{2}$ In particolare, a partire dal II secolo a.C., la diffusione della cultura greca nel mondo occidentale e specificamente in quello latino determina il successo della retorica e la sua presenza nell'iter educativo e formativo delle giovani generazioni che avevano accesso agli studi (Corno, 2011; Marazzini, 2001). ${ }^{3}$ Dalla tradizione classica ricaviamo numerosi elementi ancora oggi

2. La tradizione è così resistente che molti degli elementi e delle tecniche dell' "arte del dire" individuate nel mondo greco-latino sono ancora alla base degli attuali manuali di comunicazione.

3. Basti pensare a opere come I'Institutio oratoria di Quintiliano (c.a. 35/40 - 96): 12 volumi rivolti a educare il futuro oratore, a cominciare dall'infanzia, tramite le tecniche e l'esperienza della migliore tradizione retorica. 
significativi da considerare nella composizione di qualsiasi testo, come l'inventio (il reperimento dei contenuti), la dispositio (lo schema del discorso, I'ordine degli argomenti) e l'elocutio (lo stile, con le sue possibilità espressive che influiscono sulla comunicazione).

Nei secoli, ad affermarsi e ad affinarsi sono stati soprattutto gli aspetti legati all'elocutio e nella fattispecie all'ornatus, cioè al come dire le cose e alle figure retoriche (di cui si trovano ricchissime e dettagliate tassonomie in studi recenti come Lausberg, 1949/1969, Gruppo $\mu$, 1970/1976). Gli eccessi in questo senso hanno portato allo scadimento di certa retorica e, al suo interno, di alcune modalità argomentative, soprattutto di quelle finalizzate a far prevalere opinioni e punti di vista. Basti pensare all'irrigidimento, anche in didattica, di certi schematismi che non favorivano lo sviluppo del pensiero, ma si appiattivano sulla ripetizione stereotipa di strutture codificate: ne sono esempio estremo le cosiddette macchine retoriche in voga nella manualistica del '500 (una delle più note è quella proposta da Francesco Alunno nella Fabrica del mondo del 1548), che prevedevano schemi combinatori di parole da cui attingere per strutturare discorsi eloquenti. E una simile tendenza all'imitazione e all'appiattimento delle strategie argomentative in didattica si trova confermata anche in ambito matematico almeno fino ai primi decenni del Novecento, quando, anche tramite le nette posizioni antiretoriche dell'idealismo crociano, tutta questa tradizione viene messa in discussione. Essa verrà ripresa, in termini nuovi e critici, solo nella seconda metà del secolo sulla base di nuovi paradigmi linguistici e culturali. Questi paradigmi tentano in primo luogo di guardare all'argomentazione da un punto di vista organico, nel quale convergono tanto la tradizione retorica quanto quella dialettica. Ecco il perché dell'espressione La nuova retorica, sottotitolo del celebre Trattato dell'argomentazione del 1958 di Perelman e Olbrechts-Tyteca: con questo titolo gli autori volevano intendere che ogni argomentazione, anche quella apparentemente più impersonale, è di tipo retorico, perché «si sviluppa in funzione di un uditorio» (Perelman \& Olbrechts-Tyteca, 1958/2013, p. 8). Studi considerati oggi di base come quello già citato di Perelman e Olbrechts-Tyteca, ma anche Toulmin (1958/1975), hanno da un lato contribuito a fondare una nuova disciplina, la teoria dell'argomentazione, i cui oggetti di studio sono «le tecniche discorsive atte a provocare o accrescere l'adesione delle menti alle tesi che vengono presentate al loro assenso» (Perelman \& Olbrechts-Tyteca, 1958/2013, p. 6); dall'altro, dal nostro punto di vista, hanno fornito la base su cui fondare le discussioni circa i collegamenti fra argomentazione in senso classico e argomentazione nell'apprendimento della matematica. Tali discussioni hanno infatti consentito ai didatti della matematica di riflettere sul ruolo del linguaggio naturale e dell'argomentazione nell'apprendimento di tecniche e di ragionamenti, ma anche, ad esempio, sul problema della possibilità e delle condizioni di un passaggio dall'argomentazione alla dimostrazione. ${ }^{4}$ Insomma, moltissimo è stato detto sull'argomentazione, tuttavia - soprattutto se si vuole tentare di affrontare l'argomento in modo almeno in parte nuovo e non troppo vincolato dalla tradizione e dal suo inevitabile peso - molto resta ancora da dire se si punta lo sguardo alla didattica (laddove resta una competenza trasversale condivisa dalle diverse discipline) per studiarne la resa in luoghi apparentemente secondari ma cruciali per la formazione degli allievi, come possono essere i libri di testo. Nel farlo, teniamo ovviamente conto del fatto che per affrontare l'argomentazione nei testi scolastici di matematica di oggi non è possibile prescindere dal collocarli nella tradizione culturale, didattica e manualistica cui appartengono, per capirne forse meglio certe scelte e certe impostazioni. ${ }^{5}$

\footnotetext{
4. Non è questa la sede per affrontare l'ampio tema del rapporto fra argomentazione e dimostrazione. Per i nostri scopi, sarà sufficiente ricordare alcuni passaggi, giacché discussioni in seno alla didattica della matematica proseguono da decenni e hanno prodotto una vasta letteratura. Può essere utile ricordare che Duval (1998) e Balacheff (1988) evidenziano distanze rispettivamente di tipo cognitivo e linguistico da un lato, di tipo sociale ed epistemologico dall'altro, fra argomentazione e dimostrazione. Queste innegabili differenze sono state poi rielaborate e integrate, ad esempio, all'interno del costrutto dell'Unità Cognitiva (Boero et al., 1996; Garuti, 2003; Mariotti, 2006; Martinez \& Pedemonte, 2014; Pedemonte, 2008), con il quale si sono voluti mettere in evidenza alcuni aspetti di continuità riguardanti in particolare la generazione, durante la produzione argomentativa, della congettura, ossia degli elementi che vengono poi utilizzati durante la costruzione della dimostrazione. 5. La prospettiva potrebbe ampliarsi se si aprisse il campo agli studi etnolinguistici (come Cardona, 2006) e etnomatematici (D'Ambrosio, 2002), e alle svariate possibilità comunicative e pragmatiche che le diverse lingue e ancor più le culture offrono, anche a livello di scelte e modalità argomentative.
} 


\subsection{Alcune caratteristiche dell'argomentazione in matematica}

Da un punto di vista etimologico, il termine "argomentazione" e il sostantivo "argomento" contengono la radice lessicale del verbo latino arguo, il cui significato principale è quello di mettere in evidenza, portare a riconoscere; ancor più precisamente, nella lingua latina ha preso forma il verbo deponente argumentor, "argomentare, dimostrare, ragionare», da cui, appunto, il nostro argomentare. ${ }^{6}$ In altre parole, I'espressione indica «il processo di "aiutare" I'interlocutore a riconoscere qualcosa fornendo (direttamente o indirettamente) una opportuna giustificazione» (Rigotti \& Greco, 2009, p. 4, traduzione degli autori). Detto ancora in altri termini, I'argomentazione trova dunque le sue radici nell'esigenza di giustificare una affermazione o una tesi.

Da questa definizione emergono già alcune caratteristiche dell'argomentazione. In primo luogo, I'argomentazione non coincide con la sola affermazione di qualcosa (cioè con l'esplicitazione di stati di cose, idee, opinioni, proposte ecc., ossia di una tesi), perché a questa va necessariamente aggiunta una giustificazione.

Ma, come afferma Duval (1998, p. 6), «nella giustificazione di una affermazione, ha importanza separare bene due operazioni: la produzione di ragioni o di argomenti, e l'esame di accettabilità degli argomenti prodotti». Dal punto di vista del loro funzionamento cognitivo, la prima operazione dipende maggiormente dalla spiegazione, la seconda dal ragionamento. Pur riconoscendo distinzioni di tipo epistemologico e cognitivo, sulle quali non entreremo in dettaglio, lo stesso Duval sostiene che le operazioni da cui è composta l'attività di giustificazione di un'affermazione siano di fatto complementari, e che spesso le giustificazioni espresse nei testi siano relativamente indifferenziate, mescolando accenni di spiegazione e accenni di argomentazione (Duval, 1998, p. 36). In quest'ottica, ci sembra giustificabile una posizione integrata come quella che assumiamo in questo contributo, e cioè di una visione nella quale un'argomentazione, soprattutto laddove viene presentata per far comprendere a qualcuno - il lettore del libro di testo e studente - un risultato matematico, può contenere al suo interno aspetti legati tanto alla spiegazione quanto all'argomentazione, in un equilibrio che può essere spostato a volte più sull'uno o sull'altra.

In secondo luogo, a livello di funzione, l'intento di un'argomentazione risulta essere la persuasione o il convincimento di diversi uditori riguardo a una o più tesi: sarà «persuasiva una argomentazione che pretende di valere soltanto per un uditorio particolare» e "convincente quella che si ritiene possa ottenere l'adesione di qualunque essere ragionevole» (Perelman \& Olbrechts-Tyteca, 1958/2013, p. 31). Dovendo influenzare il particolare uditorio a cui ci si riferisce, la persuasione fa appello anche a meccanismi soggettivi, emotivi e passionali; d'altro canto, invece, la convinzione, dovendo valere per un qualsiasi essere ragionevole, mira a «superare degli ostacoli logici e razionali con dei mezzi che hanno la parvenza della logica e della razionalità, per vincere le resistenze ed i dubbi con la forza logica delle argomentazioni» (Godino, 2009, p. 98). Queste due funzioni sono entrambe presenti nell'ambito della didattica della matematica, in particolar modo considerando i libri di testo. ${ }^{7}$ Da un lato, infatti, le argomentazioni di un libro di testo di matematica si rivolgono a un uditorio ben determinato in termini di età, e dunque anche in termini di possibilità cognitive, di capacità di attenzione, di coinvolgimento emotivo ecc.; questo comporta che chi argomenta (nel nostro caso, gli autori del libro di testo) effettui scelte il più possibile persuasive per il pubblico a cui si riferisce. Anche perché, come afferma Rousseau, convincere un bambino non serve a nulla «se non si sa persuaderlo» (Rousseau, 1898/1950, p. 171). Ad esempio, il riferimento all'esperienza reale e concreta vicina al vissuto degli allievi è particolarmente efficace dal lato della persuasione, specialmente per le fasce d'età dei più piccoli. Dall'altro lato, essendo i libri di testo oggetto di analisi di questo articolo rivolti alla matematica, disci-

6. In greco non vi era un equivalente perfetto, ma diverse forme verbali che esprimevano diverse intenzioni in contesto. 7. Poiché il contributo si occupa di argomentazioni presenti nei libri di testo di matematica, non ci siamo focalizzati sull'argomentazione in quanto discorso che si sviluppa nella pratica didattica matematica delle classi. Per una letteratura in questo senso, rimandiamo all'ampia bibliografia presente sul sito http://www.lettredelapreuve.org/. 
plina la cui natura ha un carattere logico intrinseco, la persuasione potrebbe non essere sufficiente. È facile, infatti, immaginare uno studente persuaso della validità del risultato di un'argomentazione matematica senza tuttavia esserne convinto; questo significherebbe che lo studente è disposto ad accettare il risultato, ma tale accettazione risulterebbe priva del carattere di adesione razionale proprio di chi ha compreso.

Ci sembra dunque sia importante che i libri di testo di matematica rivolti ai livelli scolastici da noi esaminati considerino entrambe le funzioni dell'argomentazione, la persuasione e il convincimento, senza dimenticare, dal lato dell'apprendimento, ciò che afferma Duval: «non è possibile convincere senza far comprendere» (Duval, 1998, p. 5). Ciò aggiunge un ulteriore elemento all'argomentazione proposta dai testi scolastici: di qualsiasi tipo essa sia (cioè quali che siano le modalità scelte e gli argomenti portati), essa, per essere efficace, deve essere compresa dai lettori così come devono essere compresi i suoi contenuti, intendendo la comprensione come processo cognitivo di interazione profonda col testo (ampiamente illustrato, per esempio, in Lumbelli, 2009), per cui è necessario mobilitare conoscenze pregresse (nel nostro caso, disciplinari), linguistiche, esperienziali ecc., e farle interagire col testo. Consideriamo tutti questi elementi e focalizziamoci sull'aspetto illocutivo (Austin, 1962/1987): poiché l'effetto desiderato dei passaggi logico-argomentativi dei libri di testo è che i destinatari siano persuasi e, poi, pian piano, razionalmente convinti di un sapere matematico, un'argomentazione scritta deve essere prodotta e ricevuta "felicemente", ossia il ricevente dovrebbe essere posto nella condizione di aderire alla tesi dell'emittente valutando la tesi stessa e le prove (comprendendo il linguaggio, attivando le conoscenze necessarie, facendo interagire diversi elementi richiamati ecc.). Insomma, come ricorda Sbisà (1989), i partecipanti all'atto dovrebbero implicitamente essere d'accordo, nel senso che dovrebbero essere partecipi dell'atto stesso, cioè consapevoli e attivi, altrimenti i passaggi logico-argomentativi stessi potrebbero non essere recepiti come tali: il testo non basta, se non c'è il contributo del lettore, che, a scuola, dovrebbe gradualmente essere educato a questo tipo testuale e alle sue molteplici forme e finalità.

Per poter procedere nella nostra trattazione, occorre ora addentrarsi nella forma che assume l'argomentazione del testo scritto, che ha specifiche peculiarità pragmatico-comunicative.

\subsection{L'argomentazione nel testo scritto di matematica: la diamesia e la prospettiva funzionale}

Come si è intuito dall'ampiezza del quadro teorico, parlare di argomentazione significa muoversi in un territorio vasto, con alcuni elementi costanti e ricorrenti, ma con anche svariati fattori di diversità da un'occasione a un'altra. Comunque lo si intenda, infatti, argomentare non significa solo inanellare parti di discorso (tesi, prove e così via), ma anche curarne l'efficacia per i destinatari, la «felicità» in termini pragmatici. Cosa che può differire non poco da un testo a un altro e da una situazione a un'altra.

Prima di passare a esempi di analisi, risulta dunque significativo richiamare almeno una dimensione fondamentale di variazione della lingua ${ }^{8}$ (anche delle lingue speciali come quella della matematica): quella della diamesia, ossia del mezzo usato per comunicare. Ciò perché le nostre analisi si riferiscono a parti di testi scritti nelle quali la dimensione esplicativa si caratterizza in termini logico-argomentativi e non ad argomentazioni che si sviluppano nell'oralità, e che potrebbero coinvolgere un'interazione diretta e immediata con un interlocutore, tramite azioni quali la discussione o il dibattito (frequenti e utili anche in didattica). In questo senso va ricordato che i libri di testo sono dispositivi tendenzialmente rigidi dal punto di vista non solo dell'interpretazione, ma anche dell'interazione comunicativa: in essi - non essendoci alcun processo di negoziazione immediata di significato fra interlocutori -, la ricostruzione del significato è a carico esclusivo del lettore (P. L. Ferrari, 2004).

8. Il riferimento è ai classici lavori di Berruto (1987) in cui sono illustrate le dimensioni di variazione linguistica. 
Da questa specificità di mezzo fisico-ambientale attraverso cui si svolge l'atto comunicativo derivano, ovviamente, caratteristiche linguistiche ed espressive diverse, e dunque una pragmatica specifica, in quanto la lingua scritta non è semplicemente il parlato trasferito su pagina, ma presenta proprie peculiarità. Come spiega Serianni $(2003,2010)$, alcune differenze sostanziali lo contraddistinguono:

- lo scritto si avvale prevalentemente del canale grafico-visivo (mentre il parlato utilizza il canale fonico-acustico): ciò è ben visibile in passaggi di trattamento e conversione tra un registro semiotico e un altro, fondamentali per l'apprendimento in ambito matematico;

- lo scritto è solo eccezionalmente "in situazione", mentre più spesso si scrive a un destinatario distante o a uno o più destinatari ideali, mentre, per contro, il parlato è tipicamente "in situazione" e presuppone un emittente che si rivolge a dei destinatari che possono interagire nel discorso (se le circostanze lo permettono);

- lo scritto è rigido, sequenziale e non dà possibilità di feedback immediati. Per contro, in un dialogo chi parla può considerare le reazioni dell'interlocutore o anche le sue difficoltà o il suo disinteresse;

- lo scritto è fruibile liberamente dal destinatario, senza obbligo di svolgimento lineare: in moltissimi casi di un testo si leggono solo le parti che interessano (cosa frequentissima nei libri di testo);

- lo scritto, almeno quello più formale e controllato, è regolato e rigido, mentre il parlato può essere in una certa misura maggiormente ambiguo e sollecitare di più la cooperazione dell'ascoltatore.

Tenendo sullo sfondo questi elementi, riteniamo interessante guardare alle scelte argomentative effettuate nei libri di testo richiamando anche la classificazione funzionale dei testi, di cui quello "argomentativo" costituisce un tipo. Dal punto di vista linguistico-testuale, I'organizzazione dei testi per tipi funzionali - originariamente impostata da Werlich (1982) - prevede che il tipo argomentativo si distingua da quelli narrativo, descrittivo, espositivo e regolativo per alcuni tratti salienti (Lala, 2011; Mortara Garavelli, 1988). Come scrive Colombo (1992, p. 477) traducendo Werlich, in generale nel testo argomentativo «il codificatore propone relazioni tra concetti o fenomeni», e, come specifica $A$. Ferrari (2019, p. 82), pur condividendo alcune caratteristiche essenziali, «ogni testo argomentativo presenta [...] una sua struttura specifica, tanto a livello logico-semantico che a quello referenziale». Ciò significa che i contenuti possono essere i più vari e presentati in modi diversi e secondo diverse strategie, ossia attraverso una particolare organizzazione logico-semantica: ad esempio il ragionamento presentato può essere di tipo induttivo (dal particolare al generale), oppure deduttivo (dal generale al particolare), modalità entrambe presenti nei testi di matematica.

Ad ogni modo, per dirsi argomentativo, un testo o una parte di testo deve presentare alcuni tratti fondamentali (mentre altri non sono obbligatori né lo è l'ordine in cui compaiono): dovrà avere una tesi (un'idea, un'asserzione), uno o più argomenti a sostegno della tesi e una o più regole generali che garantiscano il nesso fra tesi e argomenti. Ovviamente, i Movimenti Testuali logico-argomentativi del testo scolastico di matematica non rappresentano che un sottotipo molto specifico e particolare di argomentazione; in questo senso, va richiamato quanto scrive Cortelazzo (1994, p. 4) riprendendo Dressler e Beaugrande (1984): i testi scientifici e soprattutto, pensando alle nostre analisi, alcune loro parti, sono ascrivibili al tipo argomentativo ricorrendo a "un'accezione larga di questa etichetta», intesa come caratteristica di un testo che accompagna il lettore nella costruzione e contemporaneamente nella comprensione di idee e concetti, consolidandone l'interiorizzazione tramite prove di varia natura.

Molti altri elementi che sono stati sviluppati dagli studi sulla testualità argomentativa possono essere considerati per vedere come si manifestano nei libri di testo: il tipo di fonti selezionate, il ricorso a esempi come base sulla quale operare congetture allo scopo di costruire concetti, I'uso di controesempi o di non-esempi che portano a un conflitto di punti di vista e conducono a un confronto di 
argomenti i quali giustificano affermazioni contradditorie o affermazioni contrarie su una questione. È interessante notare come queste tecniche argomentative siano molto presenti nella pratica matematica, e, soprattutto in ambito geometrico, siano utilizzati prevalentemente come supporti euristici al ragionamento. Del ruolo fondamentale dell'esempio, del non-esempio e del controesempio nella produzione di argomentazioni matematiche parlano numerosi studi a livello internazionale (Antonini et al., 2011; Balacheff, 2001; Buchbinder \& Zaslavsky, 2011; Pedemonte \& Buchbinder, 2011; Watson \& Mason, 2006), ma va considerato che nei libri di testo sono presenti quasi esclusivamente gli esempi sui quali si basano quasi tutte le argomentazioni previste per questi livelli scolastici e in rari casi i non-esempi (cioè, per menzionarne uno, il cerchio come non-esempio di poligono), mentre non sono presenti controesempi.

Nel prossimo paragrafo presenteremo alcuni elementi linguistici inerenti specifici connettivi, che sfrutteremo in fase di analisi.

\subsection{I connettivi e altre "spie linguistiche" nell'argomentazione}

Di recente, i lavori sull'argomentazione dal punto di vista linguistico si sono fatti via via più fini, recuperando ma soprattutto arricchendo presupposti antichi. Ad esempio, alcuni studi di argomentazione degli ultimi decenni, come Lo Cascio (1991) e lacona (2005), si soffermano sulla struttura argomentativa, sull'articolazione e sulla forza degli argomenti, e sull'uso dei connettivi, o «indicatori di forza» (così sono chiamati in Lo Cascio, 1991). Oltre alla scelta e alla concatenazione dei contenuti, anche alcune "spie linguistiche", variamente collocate nello snodarsi dell'argomentazione, possono infatti conferirle forza, ossia efficacia, cooperando a rendere «felice» (Austin, 1962/1987), per dirla in termini pragmatici, I'atto comunicativo. «Felice» nel senso che ottiene il risultato auspicato e diventa operativo nel contesto: nel nostro caso, significa che l'atto è costruito per presentarsi in modo efficace ai giovani lettori che stanno acquisendo concetti matematici e, al contempo, stanno prendendo dimestichezza con varie modalità comunicative disciplinari.

L'interesse per lo studio dei connettivi utilizzati nelle argomentazioni è condiviso anche dalla ricerca in didattica della matematica. Nel già citato contributo di Duval (1998), ad esempio, l'autore si domanda se sia possibile riconoscere, nella presenza di connettivi, criteri per identificare la presenza di un'argomentazione. Egli identifica tre tipi di connettivi: ${ }^{9}$ i connettivi combinatori, che integrano più proposizioni in una sola superproposizione il cui valore di verità dipende dalle proposizioni di cui è costituita (ne sono esempi il se... allora, la o esclusiva, la o, la e, molto utilizzati in matematica); i connettivi argomentativi, per Duval tipici del discorso argomentativo, che mettono in rapporto due proposizioni ma non le integrano in una superproposizione, e che possono essere distinti in connettivi di co-orientamento (anche), e in connettivi di contro-orientamento (ma, anche se, benché, tuttavia,...); infine i connettivi organizzativi, per Duval tipici della spiegazione, che indicano lo statuto di una proposizione in rapporto ad altre proposizioni, determinando quindi il suo posto nell'organizzazione del discorso. Quest'ultimo tipo di connettivi, in particolare, consente di distinguere all'interno di ogni argomentazione quali proposizioni fungono da premesse, termini medi e conclusioni: di conseguenza, quindi, dunque, perciò (collegano la tesi se questa segue gli argomenti); si sa che, in base a (introducono la regola generale); perché, poiché, infatti, considerato/visto che (introducono gli argomenti); tranne che, a meno che (introducono una riserva, cioè la possibilità che esistano dati ed elementi che conducono a conclusioni diverse). Nonostante la presenza di questo tipo di connettivi

9. Esistono in linguistica molte classificazioni dei connettivi, più o meno vaste e finalizzate a mettere in luce differenti potenzialità e funzioni di questa ampia e aperta classe di parole. In questo contributo abbiamo citato per esteso quella di Duval perché, pur condividendo alcuni elementi di fondo con altre tassonomie, orienta l'attenzione agli aspetti che maggiormente fanno comprendere il ruolo dei connettivi come organizzatori argomentativi in matematica. Per una tipologia dei connettivi come elementi che esplicitano le relazioni logiche di un testo a livello di rapporti fra gli eventi e di composizione testuale si rimanda a A. Ferrari (2014, 2019) e Ferrari e Zampese (2016). 
possa orientare nel riconoscere le peculiarità di un discorso argomentativo, lo stesso Duval mette in guardia da categorizzazioni troppo nette: se è infatti plausibile ad esempio che in un'argomentazione si ritrovino più connettivi argomentativi, è d'altra parte possibile che «certe parole o espressioni possano essere impiegate come connettivi combinatori o come connettivi argomentativi [...] Allo stesso modo alcune parole possono essere usate come connettivi argomentativi o come connettivi organizzativi» (Duval, 1998, p. 39).

Ciò che è stato finora brevemente ripercorso, ovviamente, è appannaggio della teoria dell'argomentazione, tanto in ambito linguistico quanto in ambito matematico. Nella realtà, soprattutto in quella dei testi scolastici di nostro interesse, le cose possono funzionare in modo molto diverso ed essere messe in discussione. Ad esempio, i Movimenti logico-argomentativi individuati nei testi del nostro corpus (soprattutto quelli destinati agli allievi più giovani) non per forza contengono tutti i tratti e gli elementi tipici delle movenze argomentative né si avvalgono solo delle strategie linguistiche e contenutistiche ripercorse: la cosa interessante sarà proprio addentrarsi nell'analisi per vedere le differenze, le strategie e le specificità, in modo da riuscire ad avere un quadro di che cosa possa intendersi, in senso lato, come argomentazione nel testo di matematica della scuola elementare e media, e come ciò evolva via via al progredire della scolarità verso formati più standardizzati e riconoscibili.

Fatta questa panoramica su alcune caratteristiche teoriche del tema, analizziamo quindi ora le argomentazioni secondo l'impostazione da noi adottata.

\section{Movimenti Testuali logico-argomentativi nei testi matematici per la scuola}

\subsection{Dal fare all'astrarre}

Per inquadrare la distinzione da noi scelta per i diversi tipi di Movimenti Testuali logico-argomentativi presenti nei libri del corpus, abbiamo tenuto in considerazione il passaggio avvenuto nella storia della matematica dal mondo concreto, legato al reale, al mondo astratto; evoluzione che si presenta in modo analogo nelle fasi di apprendimento degli allievi al progredire della scolarità e, di conseguenza, nelle proposte argomentative dei libri di testo. Le origini della matematica sono infatti da ricercare nelle esigenze concrete dell'uomo legate all'operare nella realtà e alla lettura del mondo circostante. È pertanto innegabile che le forme più antiche di matematica fossero legate alle necessità quotidiane, e che solo con il pensiero greco questo ancoraggio alla realtà e al fare sia andato via via ristrutturandosi, fino a determinare la caratteristica di una disciplina tendente all'astrazione, anche se mai completamente staccata dal mondo dei sensi (D'Amore \& Sbaragli, 2017).

Per quanto concerne la geometria, il rapporto tra questo ambito della matematica e il mondo fisico è molto stretto e rappresenta uno degli aspetti salienti che la caratterizzano, come afferma Enriques (1906, p. 166): «[...] il difetto dello spirito matematico [...] è di non comprendere che un pensiero, il quale si appaghi di costruzioni astratte, senza la speranza, pur vaga, di cogliere in esse il quadro di una qualche realtà, sarebbe uno sterile istrumento dialettico». Di fatto, secondo tale autore la geometria è la prima rappresentazione del mondo fisico.

È soprattutto nel periodo chiamato Crisi dei Fondamenti, a cavallo tra il XIX e il XX secolo, che la geometria diventa una disciplina sempre più affrancata da ogni riferimento al reale, senza distanziarsene mai del tutto: i criteri essenziali di validità diventano la correttezza formale del ragionamento e la coerenza di un sistema formale (per approfondire questa evoluzione storica si vedano D'Amore \& Sbaragli, 2017, 2018, 2019, 2020).

Questa esigenza del fare per poi giungere successivamente all'astrarre si riflette, come è naturale, anche nel processo di insegnamento-apprendimento della matematica. Nella teoria evolutiva elaborata dai coniugi van Hiele (1986), riguardante lo sviluppo del pensiero geometrico, sono stati individuati dei veri e propri livelli di sviluppo che vanno dalla geometria intesa come concettualizzazione dello 
spazio percettivo, riguardante l'adattamento alla realtà del mondo fisico nel quale viviamo, alla geometria come teoria formale. L'ultimo livello dello sviluppo consiste nella capacità di muoversi all'interno di un sistema ipotetico deduttivo, ovvero all'interno di una data assiomatica.

L'importanza del fare, dell'agire concretamente, del manipolare oggetti nelle prime fasi di apprendimento viene ribadita dalle scienze cognitive che hanno ormai stabilito la centralità dell'esperienza fisica e corporea nel processo di costruzione, comunicazione e apprendimento della matematica. Fra le teorie più significative in questo senso ricordiamo la teoria dell'embodied cognition secondo la quale, per la maggior parte, gli esseri umani concettualizzano i concetti astratti matematici in termini concreti, utilizzando idee e modelli di ragionamento fondati sul sistema senso-motorio che interagisce con il mondo (Lakoff \& Núñez, 2000/2005; Robutti, 2006). L'espressione embodied cognition significa letteralmente conoscenza "incorporata" o "incarnata" e rimanda all'idea che la nostra mente e i suoi processi cognitivi più complessi si basino e si costruiscano sulla fisicità e sui movimenti del corpo umano. Si può insomma affermare - e ciò non vale solo per i concetti matematici, ${ }^{10}$ che

\begin{abstract}
«una delle grandi scoperte della scienza cognitiva è che le nostre idee sono modellate dalle nostre esperienze corporee: non nella semplice modalità di corrispondenza uno a uno, ma indirettamente, attraverso la conoscenza del nostro intero sistema concettuale nella vita quotidiana. II punto di vista cognitivo ci induce a chiederci se anche il sistema delle idee matematiche sia fondato indirettamente sulle esperienze corporee, e se sì, precisamente come».
\end{abstract}

(Lakoff \& Núñez, 2000/2005, p. 14)

\title{
4.2 Una possibile lettura dei Movimenti logici-argomentativi: le prove di Balacheff
}

Il passaggio dal fare all'astrarre è dunque uno dei nodi centrali dell'apprendimento della matematica, richiamato anche da Balacheff $(1987,1988,2001)$ nell'affrontare il concetto di prova in matematica dal punto di vista della sua genesi. L'inquadratura scelta dall'autore ${ }^{12}$ risulta utile per distinguere e descrivere i movimenti logici-argomentativi dei testi scolastici, soprattutto in ambito geometrico, dato che: «[la geometria] è esemplare per la forza con la quale questo dominio particolare della matematica solleva la questione del rapporto fra conoscenze empiriche e conoscenze teoriche» (Balacheff, 2001 , p. 4). La distinzione proposta dall'autore verte sulla natura delle conoscenze in gioco e sul genere di relazioni che esse intrattengono con gli oggetti matematici proposti: abbiamo dunque ritenuto efficace per le nostre analisi rifarci a questa classificazione.

In particolare, nella sua trattazione Balacheff chiama prove pragmatiche le prove fondate sull'azione effettiva operata su rappresentazioni di oggetti matematici. Le azioni effettive sulle rappresentazioni di un oggetto geometrico possono essere sostituite dal riferimento alla rappresentazione stessa o da un'azione che prende la forma di un'esperienza mentale. In questo caso, la prova è sollecitata dal linguaggio, mezzo di evocazione delle esperienze comuni agli interlocutori. In simili prove è centrale I'ostensione ed è evidente la loro peculiarità nella didattica della matematica, che, per arrivare a oggetti astratti, non può fare altro che passare attraverso rappresentazioni concrete.

Le esperienze mentali sono una delle tappe del processo che conduce alle prove intellettuali, ossia a

10. E la prospettiva è ormai accolta in altri fondamentali ambiti acquisizionali, come quello dell'alfabetizzazione (secondo la prospettiva aperta da Ferreiro \& Teberosky, 1985), per cui per arrivare a concettualizzare l'astrazione del sistema alfabetico il bambino percorre naturalmente fasi di sviluppo che lo portano a un progressivo distacco dal reale e dall'ipotesi iniziale di una coincidenza realtà-lingua.

11. A ciò si potrebbe aggiungere, e come vedremo sarà significativo in riferimento alle parti testuali logico-argomentative proposte nei libri, un cenno al concetto di «grounded cognition» (Barsalou, 2008), che include, oltre agli stati corporei, le simulazioni e l'influsso dell'ambiente fisico e sociale in cui siamo inseriti.

12. Pur traendo origini da lavori di più di trent'anni fa, l'impostazione proposta da Balacheff è tutt'ora ritenuta di fondamentale importanza nell'ambito della ricerca in didattica della matematica, al fine di inquadrare i processi argomentativi e dimostrativi degli studenti (si vedano ad esempio Ellis et. al, 2019; Mejía-Ramos \& Weber, 2020; Miyazaki et al., 2017). 
prove staccate dall'azione ed espresse tipicamente attraverso delle condotte linguistiche che esprimono gli oggetti, le loro proprietà e le relazioni in gioco. Nelle prove intellettuali si tende ad abbandonare il cosiddetto linguaggio della familiarità, presente nelle prove pragmatiche, che porta il segno del tempo e della durata di colui che agisce e del contesto della sua azione: il linguaggio diventa sempre più decontestualizzato, dunque distante da uno specifico oggetto rispetto a cui vengono attuate azioni, per accedere a classi di oggetti; depersonalizzato, essendo separato dall'azione (potremmo anche dire deagentivizzato); detemporalizzato, svincolando le operazioni dalla loro data e dalla loro durata aneddotica (è un linguaggio assoluto, non legato a episodi e privo di cronologia). Assume, cioè, sempre di più tutti i tratti tipici del linguaggio specialistico della matematica inteso come discorso scientifico primario.

Come sostiene Balacheff, pur essendo le prove pragmatiche «reputate meno valide», non implicano in realtà meno conoscenze di quelle intellettuali e spesso non portano a meno generalità. Non essendo il passaggio tra queste due prove scontato, l'autore delinea quattro tipi di prove che seguono la genesi evolutiva fino alla prova per eccellenza, la dimostrazione: l'empirismo naif, l'esperienza cruciale, l'esempio generico e l'esperienza mentale (Balacheff, 1987).

L'empirismo naif consiste nell'assicurare la validità di un enunciato dopo la verifica su qualche caso, che rappresenta una delle prime forme di generalizzazione scelta dagli allievi e utilizzata frequentemente anche dagli studenti più grandi (Fischbein, 1982) e, come vedremo, diffusa nei libri di testo. L'esperienza cruciale designa una prova il cui risultato permette di scegliere in maniera netta tra due ipotesi; tale esperienza serve in alcuni casi per decidere tra una proposizione e la sua negazione. Questo tipo di esperienza viene utilizzata a livello didattico ma è praticamente assente nei libri di testo del corpus. L'esempio generico rappresenta la prova più diffusa nei libri di testo e

«consiste nell'esplicitazione delle ragioni della validità di un'asserzione mediante la realizzazione di operazioni o di trasformazioni su un oggetto esaminato non per sé stesso, ma in quanto rappresentante caratteristico di una classe. La formulazione isola le proprietà caratteristiche e le strutture di una classe restando legata al nome proprio e all'esibizione di uno dei suoi rappresentanti».

(Balacheff, 2001, p. 14)

Le prove che prevedono un esempio generico rappresentano uno stadio intermedio più vicino alle prove pragmatiche o a quelle intellettuali a seconda del processo di produzione della prova, e quindi dello statuto operatorio dell'esempio utilizzato. L'importanza di questo tipo di prova risulta evidente anche dall'analisi di studenti implicati nelle attività argomentative per costruire e giustificare congetture, come sottolineato anche da alcune recenti ricerche sul ruolo dell'esempio nel fare matematica (tra le altre si vedano Antonini, 2011; Antonini et al., 2011; Pedemonte \& Buchbinder, 2011). Infine, l'esperienza mentale, che invoca l'azione interiorizzata e si distanzia dalla realizzazione su un rappresentante particolare: questa prova segna il passaggio dalle prove pragmatiche a quelle di tipo intellettuale.

\subsection{Dal movimento logico-argomentativo con la modalità di far "fare" al fare "astrarre"}

Possiamo a questo punto interpretare quanto osservato nei libri di testo del corpus in base a ciò che è stato presentato in precedenza. Va precisato che il corpus è costituito da 142 libri di testo scolastici di matematica in lingua italiana della scuola elementare e media che sono stati raccolti tra quelli editi in Italia e nei cantoni italofoni della Svizzera (Canton Ticino e Canton Grigioni). Tra questi, 129 provengono dal variegato e ampio contesto editoriale italiano; dei 13 libri di testo del contesto svizzero, 7 provengono dal Canton Ticino e 6 dal Canton Grigioni. La minore presenza di libri di testo svizzeri è dovuta al fatto che, soprattutto in Canton Ticino e in modo specifico per la scuola elementare, I'utilizzo del libro di testo in ambito didattico non è diffuso. 
Nei libri di testo scolastici presenti nel corpus si è potuto rilevare che i Movimenti Testuali logico-argomentativi sfruttano tre tipi di processi cognitivi diversi tra loro, che prevedono gradi di coinvolgimento differenti:

1. Movimento Testuale con la modalità di far "fare": il lettore è chiamato a partecipare in prima persona alla costruzione del ragionamento attraverso azioni concrete di varia natura;

2. Movimento Testuale con la modalità di far "immaginare": il lettore è chiamato a immaginare azioni sugli oggetti geometrici cui ci si riferisce, senza eseguirle concretamente;

3. Movimento Testuale con la modalità di far "astrarre": il lettore è chiamato ad astrarre, ad allontanarsi dalla realtà immediata; I'attività concreta lascia il campo al pensiero astratto, per mezzo del quale si costruisce l'argomentazione.

A livello di organizzazione interna degli elementi tipici dell'argomentazione, gli esempi che presenteremo propongono tutti la tesi alla fine, come punto d'arrivo. La cosa, però, non è sempre così: seppure in misura meno frequente, la tesi può anche essere presentata all'inizio seguita da prove di varia natura.

\subsubsection{Il Movimento logico-argomentativo con la modalità di far "fare"}

Una prima modalità attraverso cui i libri di testo propongono argomentazioni si concretizza attraverso Movimenti Testuali che chiedono agli allievi di fare un'azione concreta per scoprire gradualmente e attraverso almeno una prova (di cui loro stessi diventano artefici, seppure in modo guidato) un sapere che verrà enunciato in seguito. Per essere complete, queste argomentazioni necessitano dell'azione del lettore, il quale viene appunto coinvolto attraverso consegne inserite all'interno dell'argomentazione stessa. Queste consegne possono riguardare azioni da svolgere direttamente sul libro di testo (colorare, ripassare, scrivere, tracciare, completare ecc.), in cui sono stati volutamente lasciati spazi vuoti da riempire, come se l'argomentazione fosse un «puzzle da ricostruire, puzzle per il quale mancherebbero dei pezzi da trovare prima di assemblarli» (Duval, 1998, p. 30), oppure in altri casi riguardano consegne da effettuare al di fuori del libro di testo (ritagliare, costruire, disegnare, piegare ecc.). Riportiamo di seguito un esempio che contempla entrambi i tipi di consegne.

In Figura 1 (tratta da un libro di testo di V elementare), ad esempio, dopo aver esplicitato il "Materiale occorrente" per quella che viene indicata come attività di laboratorio, vengono descritti i passi da svolgere per scoprire la numerosità degli assi di simmetria dei triangoli equilatero, isoscele e scaleno. Il materiale occorrente va a costituire l'insieme di strumenti di cui il lettore deve disporre per svolgere una significativa attività extra-testuale, cioè per realizzare una "prova" tangibile. Seguendo quanto indicato, il lettore è chiamato a procurarsi ciò che serve, disegnare i triangoli, ritagliarli, piegarli per individuare gli assi di simmetria; infine, ormai ci si auspica persuaso e forse convinto (almeno secondo le attese del testo), è invitato a tornare ad agire direttamente sul testo completando le parti sottostanti a ogni figura, per fissare il risultato matematico generale. L'argomentazione si completa e prende forma compiuta, dunque, solo alla fine e con la collaborazione dell'allievo, che, una volta effettuata la prova e riconosciutala come parte, appunto, di un'argomentazione, completa coerentemente gli enunciati (che assumono valore di tesi). 


\section{LABORATORJO}

\section{I poligoni e l'asse di simmetria interno}

Materiale occorrente: carta a quadretti, matita, righello, forbici. TRIANGOLI

1. Con righello e matita disegna sul foglio di carta un triangolo equilatero, un triangolo isoscele e un triangolo scaleno di dimensioni abbastanza grandi, poi ritagliali.

2. Considera un triangolo per volta. Per verificare se hanno assi di simmetria interni e quanti, piega a metà in modo che le due parti siano perfettamente sovrapposte.

3. Segna la piegatura, traccia gli assi di simmetria in ogni triangolo e completa le definizioni.

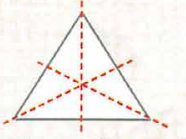

II triangolo equilatero ha 3 assi

di simmetria interni e ognuno

corrisponde a un'altezza.

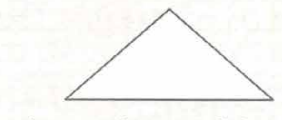

II triangolo isoscele ha asse di simmetria interno che corrisponde a un'altezza.

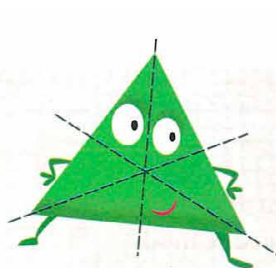

\author{
quanti,
}

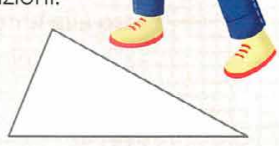

Il triangolo scaleno assi di simmetria interni.

Figura 1. Esempio di Movimento logico-argomentativo che prevede azioni concrete da svolgere fuori dal libro, su fogli, e dentro al libro, completando (libro $6 \_5$ del corpus, V elementare).

Il procedere del Movimento Testuale è semplice: a partire dall'interazione con tre triangoli specifici (un triangolo equilatero, un triangolo isoscele e un triangolo scaleno, che il lettore dovrebbe disegnare su un foglio e poi ritagliare), si generalizza il risultato ai generici triangoli equilatero, isoscele e scaleno rappresentati nelle figure (e individuati dall'articolo determinativo il con funzione generica). Ci troviamo quindi davanti a un testo che invita a realizzare una prova pragmatica, perché lo studente è chiamato a operare concretamente sulle rappresentazioni di oggetti matematici; in modo più specifico, poiché questa prova è condotta in modo empirico su tre casi di triangoli presi singolarmente, essa richiama il livello di validazione che Balacheff chiama empirismo naif: la generalizzazione del risultato ai generici triangoli equilatero, isoscele e scaleno avviene dopo la verifica su un unico caso per classe di triangoli. Dal punto di vista linguistico, il testo fa largo uso di verbi iussivi, imperativi, che esprimono un comando ("disegna", "ritaglia", "considera", "piega" ecc.), mentre la frase "Per verificare se" è utilizzata con funzione compositiva di connettivo argomentativo per collegare l'azione di piegatura del foglio alla presenza di assi di simmetria interni al triangolo. II fatto che i tre triangoli costruiti siano dei casi particolari non è introdotto da nessuna spia linguistica né vi sono connettivi che accompagnano alla generalizzazione: il «filo argomentativo» (strand, secondo Kopperschmidt, 1985) che dovrebbe generarsi fra prove particolari e asserzioni finali (le tesi) è implicito e lasciato costruire all'allievo.

Da questo caso emerge chiaramente come questa modalità di Movimento Testuale trae la sua efficacia non tanto dal proporre argomentazioni tout court, da leggere e comprendere, quanto piuttosto dallo spingere il lettore ad affiancare alla lettura del testo esperienze e atti da svolgere concretamente. In questo modo, chi legge può giungere a persuadersi del risultato, perché esso fa leva su esperienze che egli realizza personalmente, ma può anche convincersi dello stesso, poiché è portato a scoprire attivamente gli snodi logici e concettuali dell'argomentazione. Ciò mette in luce una peculiarità del libro di testo, soprattutto per i bambini e i ragazzi più giovani: se normalmente l'argomentazione è un atto che ci vede nettamente collocati in un ruolo comunicativo (o argomentiamo o siamo i destinatari di un'argomentazione), nell'esempio qui riportato il destinatario è anche compartecipe della costruzione argomentativa stessa.

\subsubsection{Il Movimento logico-argomentativo con la modalità di far "immaginare"}

Questa modalità di realizzazione di Movimenti logico-argomentativi propone all'allievo di seguire 
un'azione già svolta nel libro o di immaginare una situazione allo scopo di interiorizzare un concetto già presentato nel testo. Non si tratta dunque di un "fare" concreto, ma di immaginare la situazione proposta. L'atto dell'immaginare può essere favorito dal libro tramite diversi tipi di espedienti grafici (frecce, associazione di uso del colore, disegno delle forbici che tagliano ecc.).

In Figura 2 (tratta da un libro di testo di IV elementare) viene proposto il caso di un Movimento logico-argomentativo in cui si giunge alla formula dell'area di un rombo equiesteso a un rettangolo (del quale è prerequisito la conoscenza della formula dell'area). Nella parte di sinistra troviamo la descrizione linguistica della procedura con la quale un qualsiasi rombo può essere trasformato in un rettangolo equiesteso; nella parte di destra viene affiancata una rappresentazione grafica della procedura stessa; il tutto è seguito da un riquadro giallo, nel quale si riprende linguisticamente (in modo però più condensato) quanto appena mostrato, aggiungendo alcuni simboli e la formula simbolica dell'area del rombo.

\section{L'AREA DEL ROMBO}

Qualsiasi rombo può essere trasformato in un rettangolo. Osserva.

Se tracciamo le diagonali, il rombo resta diviso in 4 triangoli rettangoli uguali. Se poi disponiamo i triangoli in modo diverso, otteniamo un rettangolo equicomposto e quindi equivallente.

L'area di un rombo corrisponde perciò a quella di un rettangolo che ha la base lunga come la diagonale maggiore e l'altezza lunga come metà
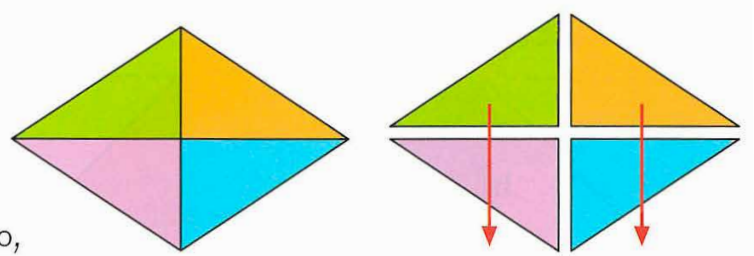
della diagonale minore.

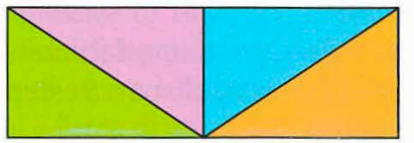

DA RICORDARE

L'area del rombo si calcola moltiplicando la misura della diagonale maggiore (D) per la misura della diagonale minore (d) e dividendo poi il risultato per 2.

Questa è la formula: $(\mathrm{D} \times \mathrm{d}): 2$

Figura 2. Esempio di Movimento logico-argomentativo che invita a immaginare attraverso l'uso di frecce (libro c3_4 del corpus, IV elementare).

Anche questa modalità di Movimento logico-argomentativo presenta elementi di similarità con le prove pragmatiche, perché in essa vengono richiamate azioni da svolgere su rappresentazioni di oggetti geometrici; essi però presentano anche caratteristiche peculiari di una validazione più avanzata, che fa riferimento a quelli che Balacheff (2001) chiama esempi generici. Rispetto alle prove pragmatiche, nell'esempio generico perde di centralità l'azione concreta svolta su un oggetto specifico: esso consiste piuttosto

«nell'esplicitazione delle ragioni della validità di un'asserzione mediante la realizzazione di operazioni o di trasformazioni su un oggetto esaminato non più per sé stesso, ma in quanto rappresentante caratteristico di una classe. La formulazione isola le proprietà caratteristiche e le strutture di una classe restando legata al nome proprio e all'esibizione di uno dei suoi rappresentanti».

(Balacheff, 2001, p. 14)

Nel Movimento testuale in Figura 2, il rombo raffigurato non è altro che un rappresentante caratteristi- 
co della classe dei rombi, e questo è particolarmente evidente se si considera l'uso del quantificatore "qualsiasi" nell'asserzione iniziale (che funge da premessa assunta come vera), che serve a chiarire che ciò che verrà detto vale per la totalità dei rombi. Nella parte testuale di sinistra troviamo numerosi altri indicatori linguistici particolarmente significativi dal punto di vista di un'argomentazione matematica. Attraverso l'uso delle protasi "se tracciamo le diagonali" e "se disponiamo i triangoli in modo diverso", si porta il lettore su un piano ipotetico, ma anche logico-deduttivo, poiché a ognuno di questi "se" corrispondono conseguenze geometriche ben precise, espresse dalle due apodosi "il rombo resta diviso in 4 triangoli rettangoli uguali" e "otteniamo un rettangolo equicomposto e quindi equivalente" (I' "allora" non è espresso, ma questi periodi sono esempi di superproposizioni). Infine, I'uso del connettivo organizzativo "perciò" indica che ci si trova di fronte alla conclusione di un ragionamento. Presa da sola, però, la parte linguistica di sinistra non è autosufficiente ai fini di un'argomentazione convincente: soprattutto non si evince dalla sola lettura del testo il modo in cui occorre disporre diversamente i triangoli che compongono il rombo per ottenere il rettangolo. Per compensare questa necessità, essa viene affiancata da una parte figurale nella parte destra della pagina, nella quale si stimola nel lettore la visualizzazione mentale dei procedimenti pratici descritti nella parte linguistica. Questa scelta è tra l'altro indicatrice di come il libro di testo abbia tra i suoi intenti non solo quello di condurre un'argomentazione corretta, ma di facilitarne la comprensione di chi legge attraverso I'utilizzo di vari mezzi semiotici necessari. In questo senso, I'argomentazione in un libro di testo scolastico possiede per forza di cose anche una natura esplicativa. I procedimenti pratici sono a tutti gli effetti già svolti dal libro di testo: il lettore deve solo riconoscerli attraverso un'attività di decodifica delle informazioni linguistiche e figurali, grazie alle quali si sostiene la ragionevolezza della conclusione.

Questa conclusione, cioè la formulazione della tesi con lo stile e i termini propri della matematica, compare nel box giallo come approdo finale. In esso non si ravvisano più riferimenti all'esempio generico, e la lingua perde completamente il carattere spontaneo e familiare per farsi formulazione scientifica. Quello che prima era "L'area di un rombo" (a intendere di uno qualsiasi fra gli infiniti rombi), qui diventa "L'area del rombo", con una preposizione articolata dal valore generico che introduce il rombo come oggetto assoluto, e le forme verbali diventano impersonali ("si calcola") e nominali (i gerundi "moltiplicando" e "dividendo"); compare da ultimo la formula simbolica.

Se volessimo schematizzare questo Movimento logico-argomentativo, dovremmo pensare che chiediamo alla mente dell'allievo di seguire grosso modo la struttura di ragionamento visibile nella colonna Funzione, con annesse caratteristiche:

\begin{tabular}{|l|l|l|l|}
\hline Testo & Funzione & Connotazione linguistica & Riferimento al disegno \\
\hline $\begin{array}{l}\text { Qualsiasi rombo può essere } \\
\text { trasformato in un } \\
\text { rettangolo. }\end{array}$ & Premessa. & Linguaggio specialistico. & No. \\
\hline Osserva. & Richiamo al lettore. & Linguaggio della familiarità. & Sì. \\
\hline $\begin{array}{l}\text { Se tracciamo le diagonali } \\
\text { [...] e quindi equivalente. }\end{array}$ & Prova. & $\begin{array}{l}\text { Linguaggio della familiarità } \\
\text { e linguaggio specialistico. }\end{array}$ & Sì. \\
\hline $\begin{array}{l}\text { L'area di un rombo } \\
\text { corrisponde perciò a quella } \\
\text { di un rettangolo che ha la } \\
\text { base lunga come la } \\
\text { diagonale maggiore e } \\
\text { l'altezza lunga come metà } \\
\text { della diagonale minore. }\end{array}$ & $\begin{array}{l}\text { Conclusione del ragiona- } \\
\text { mento. }\end{array}$ & $\begin{array}{l}\text { Linguaggio della familiarità } \\
\text { e linguaggio specialistico. }\end{array}$ & Sì. \\
\hline $\begin{array}{l}\text { L'area del rombo si calcola [...] } \\
\text { Questa è la formula: } \\
\text { (D x d):2 }\end{array}$ & Generalizzazione (o tesi). & Linguaggio specialistico. & No. \\
\hline
\end{tabular}

Tabella 1. Esempio di schematizzazione di un Movimento logico-argomentativo. 


\subsubsection{Il Movimento logico-argomentativo con la modalità di far "astrarre"}

Rispetto ai precedenti, questa modalità di Movimento Testuale logico-argomentativo tende a muoversi su un piano del tutto concettuale, senza riferimenti a oggetti concreti o ad azioni mentali da seguire concretamente; il sapere viene costruito con il lettore procedendo per passi di ragionamento che richiamano unicamente aspetti teorici della matematica. In alcuni casi permangono tratti linguistici che potrebbero richiamare alla mente del lettore azioni concrete, ma che si riferiscono in realtà a stilemi e formulazioni tipiche del più volte citato linguaggio della familiarità di Balacheff (1987), che ancora risente degli echi di quella geometria pratica da cui ha preso avvio l'avventura della matematica e che ha un'importante funzione pragmatica di avvicinare il lettore al testo; in altri casi, invece, l'argomentazione rinuncia a questi tratti linguistici per focalizzarsi esclusivamente sulle relazioni fra i concetti e sulle proprietà geometriche in gioco.

Nel Movimento logico-argomentativo mostrato in Figura 3 (tratto da un libro di testo di I media), viene proposto un esempio di strategia argomentativa più astratta delle precedenti, con la quale si vuole giungere alla tesi racchiusa nel rettangolo con bordo tratteggiato in fondo alla pagina: "La somma delle ampiezze degli angoli interni di un poligono è uguale a tanti angoli piatti quanti sono i lati meno due".

\section{Angoli interni}

Consideriamo adesso gli angoli interni ad un poligono e cerchiamo di scoprire quanto misura la loro somma.

Osserviamo che per ogni poligono è possibile costruire tanti angoli piatti quanti sono i lati del poligono considerato (Fig. 6): ogni angolo piatto è formato da un angolo interno e dal suo corrispondente esterno.

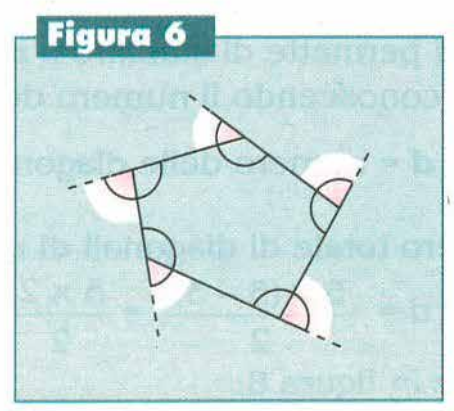

La somma degli angoli interni si ottiene quindi dalla differenza tra la somma di tanti angoli piatti quanti sono i lati del poligono e la somma degli angoli esterni, che sappiamo essere di $360^{\circ}$ qualunque sia il poligono. Indicando con $\boldsymbol{n}$ il numero dei lati possiamo scrivere:

\section{somma degli angoli interni $=n \times 180^{\circ}-360^{\circ}$}

ma un angolo giro corrisponde al doppio di un angolo piatto cioè: $360^{\circ}=2 \times 180^{\circ}$, quindi:

$$
\text { somma degli angoli interni }=n \times 180^{\circ}-2 \times 180^{\circ}=(n-2) \times 180^{\circ}
$$

N.B. Nell'ultima espressione è stata applicata la proprietà distributiva della moltiplicazione rispetto alla sottrazione, così è possibile "raccogliere" il fattore $180^{\circ}$ perché comune ai due termini della sottrazione.

In conclusione:

La somma delle ampiezze degli angoli interni di un poligono è uguale a tanti angoli piatti quanti sono i lati meno due.

Osserva che, a differenza della somma degli angoli esterni, la somma degli angoli interni non è costante ma dipende dal numero dei lati. 
Questa modalità di Movimento logico-argomentativo presenta elementi di contatto con le prove intellettuali di Balacheff, ossia prove «staccate dall'azione, inscritte nelle condotte linguistiche che esprimono gli oggetti e le loro proprietà, e che esprimono le loro relazioni» (Balacheff, 2001, p. 9). Queste prove non poggiano più su azioni da svolgere sulla rappresentazione dell'oggetto geometrico e il linguaggio «è segnato dall'introduzione di un lessico specifico e di simboli» (Balacheff, 2001, p. 11). Il punto di partenza linguistico di tale Movimento è, come si suol dire, da manuale, nel senso che ne propone i modi linguistici e le formulazioni più tipiche e ricorrenti: si dichiara che cosa si andrà ad analizzare (con verbo alla seconda plurale - almeno grammaticalmente inclusivo di chi scrive e di chi legge - molto tipico: "consideriamo adesso gli angoli interni a un poligono") e ciò che si vuole indagare ("cerchiamo di scoprire quanto misura la loro somma"). L'architettura di questo Movimento è dunque circolare: la conclusione dà la risposta alla "scoperta" annunciata. II lettore passa attraverso una fase osservativa del pentagono rappresentato in alto a destra (introdotto da "Osserviamo..."), che ha ancora una volta la funzione di esempio generico: esso è un rappresentante della classe dei poligoni e viene utilizzato per poter sostenere l'affermazione che la costruzione di tanti angoli piatti quanti sono i lati di un poligono, può essere realizzata "per ogni" poligono. Tale affermazione si snoda attraverso due enunciati separati dai due punti e connessi da una relazione di specificazione. Il verbo "costruire" utilizzato nel secondo capoverso potrebbe richiamare nel lettore un'azione concreta attraverso un verbo familiare, ma si tratta in realtà di una forma impersonale modalizzata ("è possibile costruire"), che dematerializza l'operazione, come se la lingua stessa facesse intendere che "costruire" degli angoli non può essere che un passaggio mentale, immateriale, astratto. Ciò è confermato dal fatto che si potrebbe sostituire questa formulazione della frase con un'altra in cui non compare il verbo "costruire", senza perdere nulla in termini di completezza e correttezza. ${ }^{13}$ Si riscontra in seguito il salto linguistico alla forma impersonale ("La somma degli angoli interni si ottiene...") nella parte di testo contenente anche il connettivo organizzativo "quindi", che, nel procedere argomentativo, colloca quanto viene detto in una relazione di consecuzione rispetto a ciò che precede (A. Ferrari, 2014).

Proseguendo la lettura, vengono poi esplicitati i passi di un ragionamento, in un crescendo di astrazione, che non poggia più sull'intreccio fra lingua e figura, bensì sull' uso combinato di elementi linguistici e simbolici, ad esempio nell'espressione, retta significativamente da un gerundio, "indicando con $n$ il numero dei lati". Questo enunciato porta a concludere quanto desiderato, dapprima attraverso la formula "somma degli angoli interni $=\ldots=(n-2) \times 180^{\circ}$ " evidenziata in grassetto e introdotta da "quindi", e successivamente attraverso la formulazione linguistica scritta nel rettangolo dal bordo tratteggiato, preceduta dal connettivo organizzativo "in conclusione", che colloca questa parte di testo in relazione di conclusione con le precedenti.

Il coinvolgimento del lettore con il testo è minimo: egli non è chiamato a eseguire o immaginare azioni, ma solamente a seguire, quasi esclusivamente tramite la lettura del testo scritto, il ragionamento che gli si sta proponendo. In quest'ottica, potremmo dire che si fa leva maggiormente sul carattere di adesione razionale e di convincimento piuttosto che su quegli aspetti di coinvolgimento esperienziale tipicamente più incisivi dal punto di vista della persuasione. In definitiva, in questo esempio emergono un graduale distacco dal concreto a favore dell'astrazione e un uso sempre più marcato di proprietà e simboli tipici della matematica teorica: nel nota bene, ad esempio, si dichiarano - in una relazione marginale di aggiunta e di ampliamento rispetto al resto del testo - la proprietà aritmetica (la proprietà distributiva) e la strategia aritmetica (il "raccogliere") utilizzate per derivare l'espressione simbolica voluta.

13. La frase potrebbe essere sostituita ad esempio con la seguente: "Osserviamo che ogni poligono ha tanti angoli piatti quanti sono i lati del poligono considerato". 
In questi Movimenti logico-argomentativi, il distacco dal concreto si realizza in modi e forme testuali differenti, fino a giungere al quasi completo abbandono del linguaggio della familiarità, come si nota chiaramente nel seguente esempio - in cui la struttura argomentativa è evidente -, che riportiamo senza ulteriori commenti (Figura 4):

\section{- Triangolo equilatero}

Osserviamo il triangolo equilatero inscritto in una circonferenza. Il triangolo $C E B$ è rettangolo perché l’ipotenusa coincide con il diametro e, inoltre, i suoi angoli acuti sono di $30^{\circ}$ e $60^{\circ}$. Ricordando le applicazioni del teorema di Pitagora a questo tipo di triangolo risulta:

e quindi:

$$
\begin{gathered}
\overline{C B}=\overline{E B} \times \sqrt{3} \\
\downarrow \\
\boldsymbol{l}=\boldsymbol{r} \times \sqrt{3}
\end{gathered}
$$

In generale:

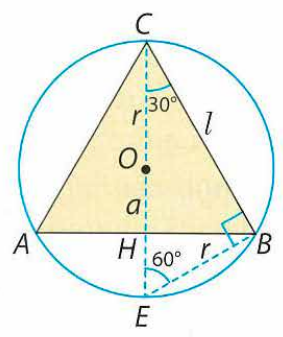

La misura del lato di un triangolo equilatero inscritto in una circonferenza è uguale alla misura del raggio per $\sqrt{3}$.

Figura 4. Esempio di Movimento logico-argomentativo che invita ad "astrarre" (libro 13_7 del corpus, II media).

\section{L'evoluzione dei Movimenti Testuali logico-argomentativi nel corpus}

Il lavoro sistematico di ricognizione di tutti i Movimenti Testuali presenti nei libri di testo del corpus ci ha permesso di profilare alcune linee di tendenza nell'uso delle modalità argomentative individuate nei paragrafi precedenti al crescere del livello di scolarità. Poiché vi sono differenze legate alla presenza e all'uso dei libri di testo nella pratica didattica tra il contesto italiano, il contesto del Canton Grigioni e quello del Canton Ticino, ${ }^{14}$ tratteremo i dati separatamente, concentrandoci dapprima sulla situazione italiana e successivamente su quella dei due cantoni svizzeri di lingua italofona.

\subsection{L'evoluzione delle modalità argomentative nel corpus italiano}

La seguente tabella (Tabella 2) mostra le quantità complessive dei diversi tre tipi di Movimenti logico-argomentativi, espresse in forma numerica e percentuale, presenti nei libri di testo italiani del corpus (129 libri di testo in totale, di cui 83 di scuola elementare e 46 di scuola media):

14. Va precisato che in Svizzera ogni Cantone è autonomo nella gestione delle scelte scolastiche; tuttavia negli ultimi anni sono stati sviluppati dei piani di studio linguistico-regionali per la scuola dell'obbligo: i Cantoni della Svizzera francese hanno elaborato il Plan d'études romand (PER, https://www.plandetudes.ch/), i Cantoni della Svizzera tedesca e plurilingui, tra i quali figura anche il Canton Grigioni, il Lehrplan 21 (Piano di studio 21, https://gr-i.lehrplan.ch/index.php) e il Canton Ticino ha elaborato il Piano di studio della scuola dell'obbligo ticinese (www.pianodistudio.ch). 


\begin{tabular}{|l|l|l|l|}
\hline $\begin{array}{l}\text { Modalità di Movimenti } \\
\text { logico-argomentativi }\end{array}$ & $\begin{array}{l}\text { Quantità nel corpus } \\
\text { italiano }\end{array}$ & $\begin{array}{l}\text { Quantità nella scuola } \\
\text { elementare }\end{array}$ & $\begin{array}{l}\text { Quantità nella scuola } \\
\text { media }\end{array}$ \\
\hline Modalità di far "fare" & $301 / 21,8 \%$ & $284 / 60,8 \%$ & $17 / 1,9 \%$ \\
\hline Modalità di far "immaginare" & $541 / 39,1 \%$ & $176 / 37,7 \%$ & $365 / 39,8 \%$ \\
\hline Modalità di far "astrarre" & $541 / 39,1 \%$ & $7 / 1,5 \%$ & $534 / 58,3 \%$ \\
\hline Totale & $1383 / 100 \%$ & $467 / 100 \%$ & $916 / 100 \%$ \\
\hline
\end{tabular}

Tabella 2. Distribuzione delle modalità di Movimenti logico-argomentativi nel corpus di libri italiani.

Globalmente sono stati individuati 1383 Movimenti testuali di tipo logico-argomentativo su un totale di 5783 Movimenti Testuali (comprendenti anche le altre tipologie esposte nel par. 2), corrispondente al 23,9\%. I Movimenti logico-argomentativi presenti in quantità maggiore sono in ugual numero i Movimenti con la modalità di far "immaginare" e i Movimenti con la modalità di far "astrarre" (541, il 39,1\%), mentre i Movimenti con la modalità di far "fare" risultano essere i meno presenti (301, il 21,8\%). L'ex aequo tra le modalità di far "immaginare" e far "astrarre" è a tutti gli effetti una coincidenza, come emerge se si considera la ripartizione dei dati fra scuola elementare e scuola media (Tabella 2). Questa ripartizione consente anche di notare il brusco cambiamento nelle percentuali delle modalità di far "fare" e di far "astrarre" nel passaggio tra scuola elementare e scuola media: la prima modalità passa dal 60,8\% all'1,9\%; la seconda, per contro, passa dall'1,5\% al 58,3\%. Per approfondire meglio questo e altri aspetti, è significativo analizzare l'evoluzione delle tre modalità di tipi di Movimento lungo tutti gli anni di scolarità considerati, dalla II elementare alla III media. ${ }^{15}$ Mostriamo, dunque, ancora più nel dettaglio, questa evoluzione attraverso una tabella (Tabella 3) e un grafico a colonne (Figura 5):

\begin{tabular}{|c|c|c|c|c|c|c|c|}
\hline \multirow{2}{*}{$\begin{array}{l}\text { Modalità di } \\
\text { Movimenti } \\
\text { logico- } \\
\text { argomentativi }\end{array}$} & \multicolumn{7}{|c|}{ Quantità nel corpus italiano } \\
\hline & II SE & III SE & IV SE & V SE & I SM & II SM & III SM \\
\hline $\begin{array}{l}\text { Modalità di } \\
\text { far "fare" }\end{array}$ & $22 / 88,0 \%$ & $51 / 86,4 \%$ & $127 / 52,1 \%$ & $84 / 60,4 \%$ & $15 / 3,0 \%$ & $2 / 0,5 \%$ & $0 / 0 \%$ \\
\hline $\begin{array}{l}\text { Modalità di far } \\
\text { "immaginare" }\end{array}$ & $3 / 12,0 \%$ & $8 / 13,6 \%$ & $116 / 47,5 \%$ & $49 / 35,3 \%$ & $235 / 47,1 \%$ & $124 / 31,7 \%$ & $6 / 23,1 \%$ \\
\hline $\begin{array}{l}\text { Modalità di } \\
\text { far "astrarre" }\end{array}$ & $0 / 0 \%$ & $0 / 0 \%$ & $1 / 0,4 \%$ & $6 / 4,3 \%$ & $249 / 49,9 \%$ & $265 / 67,8 \%$ & $20 / 76,9 \%$ \\
\hline Totale & $25 / 100 \%$ & $59 / 100 \%$ & $244 / 100 \%$ & $139 / 100 \%$ & $499 / 100 \%$ & $391 / 100 \%$ & $26 / 100 \%$ \\
\hline
\end{tabular}

Tabella 3. Distribuzione delle tre modalità di Movimenti logico-argomentativi nei libri di testo italiani dalla II SE (scuola elementare) alla III SM (scuola media).

15. D'ora in poi utilizzeremo gli acronimi SE e SM per indicare rispettivamente scuola elementare e scuola media. 


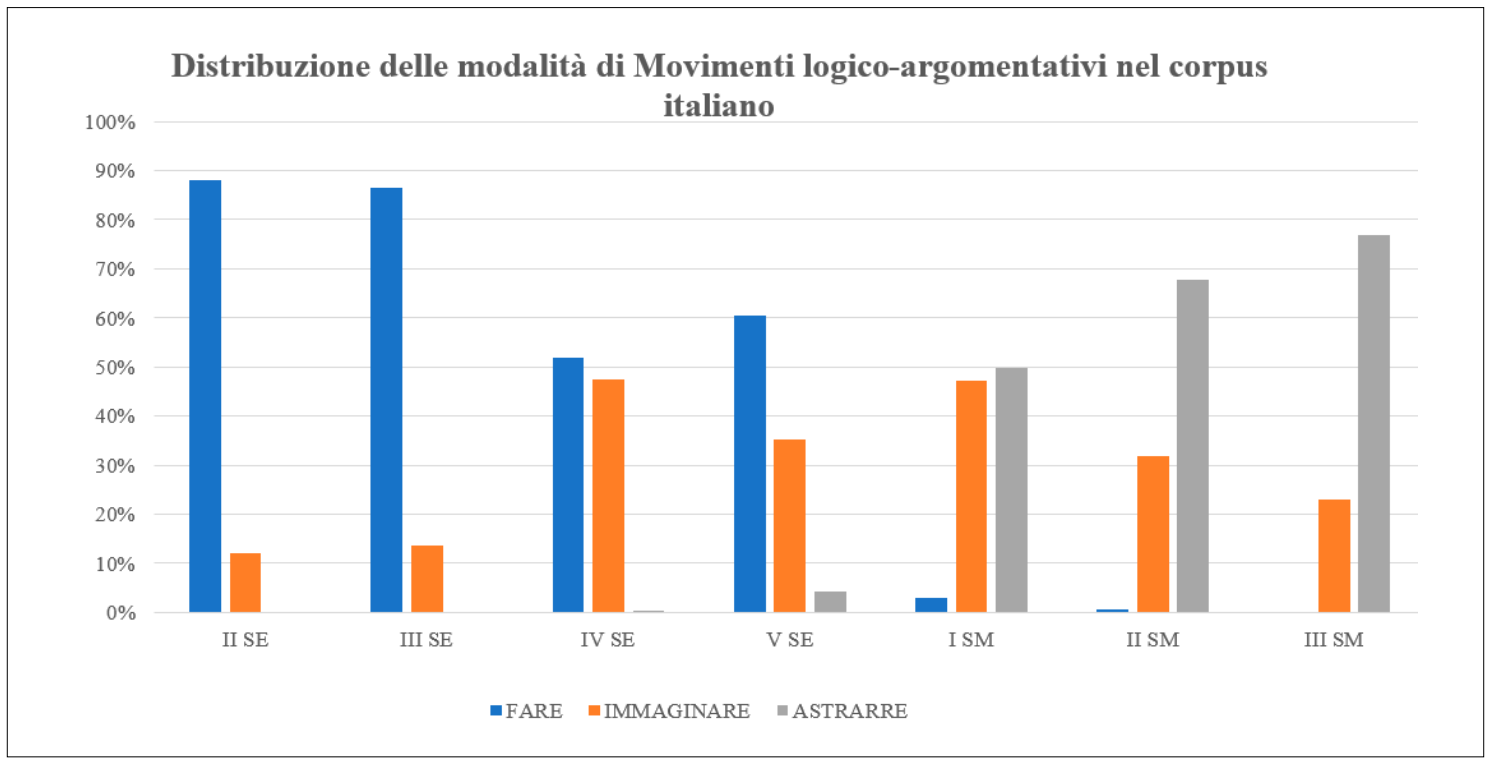

Figura 5. Distribuzione in forma di grafico a colonne delle tre modalità di Movimenti logico-argomentativi nei libri di testo italiani dalla II SE alla III SM.

In primo luogo, dal grafico emergono due tendenze globali interessanti: i Movimenti con la modalità di far "fare" - come da prassi tipiche dei testi - decrescono drasticamente all'aumentare dell'anno di scolarità, passando dall' $88,0 \%$ in II SE allo $0 \%$ in III SM. Al contrario, invece, i Movimenti con la modalità di far "astrarre" crescono all'aumentare dell'anno di scolarità, passando dallo $0 \%$ al $76,9 \%$ nelle stesse classi. Questi due andamenti opposti non sono inattesi: nei primi anni di scolarità è comprensibile che la didattica prediliga l'esperienza concreta e l'azione pratica rispetto al ragionamento astratto, mentre con l'aumentare dell'età biologica e cognitiva di chi apprende si tenda a presentare la disciplina nelle sue componenti più astratte, tipiche dei modi di ragionare e dei simbolismi propri della matematica. Per quanto riguarda l'evoluzione dei Movimenti logico-argomentativi con la modalità di far "immaginare", invece, si nota una certa costanza nel ricorso a questa strategia. Tutti questi risultati vanno letti tenendo in considerazione l'argomento di analisi scelto, il tema dei poligoni, che viene riproposto di classe in classe con ripassi e approfondimenti concettuali e di forma.

Da un'analisi più fine emerge anche come le evoluzioni delle modalità di Movimenti logico-argomentativi presentino forti tratti di discontinuità in coincidenza dei cambi di adozione dei libri di testo e del cambio di ordine scolastico (passaggi dalla III alla IV elementare e dalla V elementare alla I media); discontinuità sulla quale è importante riflettere, perché difficilmente giustificata e supportata da un'effettiva evoluzione cognitiva e di maturità degli allievi nell'arco di un'estate. Per quanto riguarda la modalità logico-argomentativa che fa "fare", si nota un brusco calo delle percentuali tra la III SE e la IV SE: dall' $86,4 \%$ al 52,1\%; crollo, ancora più marcato, nella transizione dalla V SE alla I SM: dal 60,4\% al $3,0 \%$. Considerando invece l'evoluzione della modalità logico-argomentativa che fa "immaginare", si nota un deciso aumento di percentuale nel passaggio dalla III SE (13,6\%) alla IV SE $(47,5 \%)$, come se anche in questo caso si presupponesse un rapido balzo in avanti nella capacità di staccarsi via via dal reale. Per quanto riguarda infine la modalità logico-argomentativa che fa "astrarre", nella sua evoluzione anno per anno si nota una discontinuità marcata nella transizione dalla V SE alla I SM, in cui si passa dal 4,3\% al 49,9\%: è proprio a questo riguardo che la didattica e i suoi strumenti meriterebbero una riflessione critica.

C'è da chiedersi se simili stacchi tra queste modalità logico-argomentative: far "fare", far "immaginare", far "astrarre" (dalla III alla IV SE e tra la V SE e la I SM), che risultano ormai sedimentate nelle prassi dei testi, siano davvero sostenibili per gli allievi e utili per una didattica che voglia costruire 
apprendimento e competenze; cambiamenti di modalità di ragionamento troppo bruschi potrebbero infatti concorrere all'allontanamento dalla disciplina e rendere via via più difficoltoso acquisirne i contenuti. Se si richiama la prospettiva piagetiana secondo cui il pensiero diviene pienamente formale, ossia consente di condurre ragionamenti senza la necessità di partire da un dato di esperienza, intorno ai 12 anni d'età (Lawson \& Renner, 1975; Piaget, 1972), allora anche le scelte connesse alle modalità logico-argomentative meritano una riflessione in termini di gradualità e di evoluzione, in stretto dialogo con le percezioni e con le potenzialità degli allievi, soprattutto di quelli il cui pensiero rimane più a lungo ancorato alla concretezza.

\subsection{L'evoluzione delle modalità argomentative nel corpus svizzero}

Per quanto riguarda il contesto svizzero, la ricognizione dei libri di testo utilizzati nella scuola in Canton Ticino e in Canton Grigioni ha portato ad avere 13 libri in totale (5 della scuola elementare e 8 di scuola media). Va considerato che, pur essendo un campione piccolo, esso rispecchia l'elenco complessivo dei libri in lingua italiana consigliati dagli esperti di matematica per questi Cantoni. Sono 54 i Movimenti logico-argomentativi presenti in totale su tale corpus rispetto a 604 Movimenti Testuali complessivi, corrispondente al 8,9\%. Pur trattandosi di contesti editoriali e scolastici completamente differenti, per cui è opportuna una certa cautela nel trarre conclusioni, si nota che, globalmente, la presenza di questo tipo di Movimento testuale è abbastanza inferiore nei libri in lingua italiana della Svizzera rispetto a quella presente nei libri dell'Italia (in cui, ricordiamo, la percentuale è 23,9\%).

Analizzando più in dettaglio quanto avviene nei due Cantoni, va considerato che in Canton Ticino non vengono utilizzati libri di testo nelle scuole elementari, dunque il corpus è costituito da 7 libri di testo di scuola media: 3 di I SM, 3 di II SM e 1 di III SM, corrispondenti a tre titoli diversi, e rappresentano la totalità dei libri in cui si tratta il tema dei poligoni. La seguente tabella (Tabella 4) mostra le quantità dei tre tipi di Movimenti logico-argomentativi presenti in questi testi, espressa in forma numerica e percentuale:

\begin{tabular}{|l|l|}
\hline Modalità di Movimenti logico-argomentativi & Quantità nel sub-corpus ticinese \\
\hline Modalità di far "fare" & $21 / 40,4 \%$ \\
\hline Modalità di far "immaginare" & $12 / 23,1 \%$ \\
\hline Modalità di far "astrarre" & $19 / 36,5 \%$ \\
\hline Totale & $52 / 100 \%$ \\
\hline
\end{tabular}

Tabella 4. Distribuzione delle modalità di Movimenti logico-argomentativi nel sub-corpus di libri ticinesi.

Come emerge dai dati, le modalità di Movimenti logico-argomentativi in Canton Ticino sono distribuite diversamente rispetto a quelli di scuola media italiani, avendo una prevalenza di modalità che fanno "fare" (21 Movimenti, pari al 40,4\%) rispetto alle altre due. Se poi si analizza l'evoluzione delle tre modalità lungo tutti gli anni di scolarità, dalla I alla III SM, emergono alcuni altri aspetti interessanti (Tabella 5): 


\begin{tabular}{|l|l|l|l|} 
Modalità di Movimento & \multicolumn{3}{|l|}{ Quantità nel sub-corpus ticinese espresse in forma numerica e percentuale } \\
\cline { 2 - 4 } logico-argomentativo & I SM & II SM & III SM \\
\hline Modalità di far "fare" & $10 / 50,0 \%$ & $11 / 61,1 \%$ & $0 / 0 \%$ \\
\hline Modalità di far "immaginare" & $8 / 40,0 \%$ & $3 / 16,7 \%$ & $1 / 7,1 \%$ \\
\hline Modalità di far "astrarre" & $2 / 10,0 \%$ & $4 / 22,2 \%$ & $13 / 92,9 \%$ \\
\hline Totale & $20 / 100 \%$ & $18 / 100 \%$ & $14 / 100 \%$ \\
\hline
\end{tabular}

Tabella 5. Distribuzione delle tre modalità di Movimenti logico-argomentativi nei libri di testo ticinesi dalla I SM alla III SM.

La modalità logico-argomentativa che fa "immaginare" sembra seguire una tendenza globale di decrescita graduale che si evidenzia sia nel passaggio dalla I SM alla II SM (dal 40,0\% al 16,7\%), sia dalla II SM alla III SM (dal 16,7\% al 7,1\%). Inoltre, si notano due bruschi cambiamenti nel passaggio dalla II alla III SM: scompare la modalità logico-argomentativa che fa "fare" (dal 61,1\% allo 0,0\%), mentre si impone la modalità logico-argomentativa che fa "astrarre" (dal 22,2\% al 92,9\%). In questo caso sembra che i libri ticinesi ricalchino la stessa mancanza di gradualità riscontrata nel caso dei libri italiani, ma questa volta spostata due anni in avanti rispetto a questi ultimi, rispettando dunque maggiormente i tempi di maturazione dell'età cognitiva degli allievi. Per il salto relativo ai Movimenti logico-argomentativi che avviene dalla II alla III SM occorre ricordare che il tema viene affrontato a spirale nei diversi anni e in III SM viene affrontato come ripasso dei saperi già appresi negli anni precedenti.

Al di là dei dati quantitativi e di cambiamento negli anni, a livello qualitativo i Movimenti logico-argomentativi dei libri prodotti nei due diversi contesti nazionali sono analoghi e non mostrano particolari differenze di realizzazione. Va però almeno citata, a titolo di curiosità per la sua originalità, la scelta dell'editore Casagrande (editore di 5 dei 7 libri considerati) di avvalersi nei suoi testi di un andamento dialogico a cui non è estranea la storia della manualistica in lingua italiana, soprattutto in ambito linguistico. Ne deriva che anche i passaggi logico-argomentativi, talvolta, possono trovarsi inseriti in questo tipo di andamento trattatistico, con esiti come questo (Figura 6):

A proposito di angoli, devi ricordare una caratteristica importante di tutti i triangoli:

la somma degli angoli interni di un triangolo è sempre di $180^{\circ}$ (corrisponde cioè a un angolo piatto).

Gero: "Io ho verificato questa particolarità disegnando e ritagliando parecchi triangoli, ai quali strappavo poi gli angoli, che incollavo ordinatamente in modo che risultassero consecutivi.»

Aba: "Io, invece, ho misurato gli angoli di alcuni triangoli e ho verificato che la somma dava sempre $180^{\circ}$, pur tenendo conto di piccoli errori dovuti all'imprecisione delle misure con il goniometro. »

Figura 6. Esempio di Movimento logico-argomentativo che invita a "immaginare" (libro T1_6 del corpus, prima media). 
Qui il Movimento logico-argomentativo, che negli esempi precedenti era sempre presentato dal libro al lettore in forma statica, prende vita nelle parole dei due personaggi-guida del testo: la tesi enunciata - "la somma degli angoli interni di un triangolo è sempre di $180^{\circ}$ (corrisponde cioè a un angolo piatto)" - è sostenuta dalle prove di disegno, ritaglio e misura resocontate in forma di discorso diretto dalle voci dei due; al lettore non resta che seguirle e simularle con la mente (non vi sono immagini annesse).

Per quanto riguarda il Canton Grigioni, infine, occorre chiarire preliminarmente che le scuole elementari durano sei anni, e non cinque come avviene in Canton Ticino e in Italia; inoltre, per ogni anno scolastico vi è un unico libro di testo di matematica in lingua italiana utilizzato in ambito scolastico. In totale sono stati dunque raccolti 5 libri di scuola elementare (dalla II SE alla VI SE) e un libro di scuola media (I SM), mentre nelle successive classi di SM non è presente questo argomento. Nei libri di testo delle elementari non sono stati trovati Movimenti logico-argomentativi, mentre nel libro di I SM si è riscontrata unicamente la presenza di due Movimenti logico-argomentativi con la modalità di far "fare". Si notano dunque scelte diverse di tipi di Movimenti Testuali a seconda del contesto culturale.

\section{Riflessioni conclusive in prospettiva didattica}

È oggi noto che le competenze comunicative e argomentative sono considerate centrali e fondamentali in prospettiva educativa dalle diverse discipline. Detto in termini più semplici, sapersi spiegare, sapere fornire prove e motivazioni a sostegno di ciò che si pensa o di ciò che si è fatto (ad esempio di un processo risolutivo di un problema), saper cogliere e comprendere un'argomentazione efficace (e, per contro, una non efficace o contenente fallacie) e produrre un testo (orale o scritto) coerente, coeso e ben organizzato per questi fini sono attese alte, ma centrali nel mondo scolastico e non solo. Resta tuttavia ancora poco esplorata la riflessione relativa a come lavorare su queste competenze in modo graduale, profondo e davvero efficace sul lungo termine. Se si considera che quella ad argomentare è sia un'abitudine mentale trasversale, sia un'azione concreta con specificità a seconda delle discipline, dei contesti, degli scopi ecc., allora una visione il più possibile globale delle occasioni di esposizione all'argomentazione (in senso lato) e di esercizio della stessa a scuola sin dai primi anni è un passo importante per rendersi conto delle debolezze, dei punti di forza e delle strategie più proficue. In questa visione, anche quel particolare strumento che sono i libri di testo di matematica offre uno spiraglio di indagine molto promettente, se si considera che nel campo della didattica della matematica la costruzione di competenze argomentative è essenziale, e deve essere sostenuta da efficaci competenze linguistiche generali e specialistiche.

Ma quali strumenti e occasioni diamo agli allievi per costruire tali competenze? I libri sono d'aiuto oppure continuano a parlare la loro lingua senza chiedersi se i modi che propongono sollecitano e attivano davvero i lettori? Quanto sono efficaci in termini di consapevolezza e metacognizione rispetto al macro-atto argomentativo? E poi ancora: le diverse modalità descritte e la loro evoluzione al procedere della scolarità sono realmente adeguate allo sviluppo cognitivo dei bambini e dei ragazzi e alle loro esigenze? Simili interrogativi sono emersi nello studio da noi effettuato.

L'analisi dei libri di testo ha mostrato come esistano tre modalità principali con i quali il testo propone argomentazioni al lettore. Queste tre modalità cambiano con l'evolvere degli anni di scolarità, passando dal prediligere il fare nei primi anni delle elementari fino a giungere a gradi di astrazione marcati alle medie. Per quanto riguarda il contesto italiano, la mancanza di gradualità con la quale avvengono questi passaggi sembra significativa. Se, infatti, da un lato il libro di testo non può essere in generale considerato come l'unico supporto utilizzato dagli insegnanti per strutturare e organizzare i contenuti da trattare in classe, bisogna d'altro canto ammettere che esso è uno dei mediatori che influenzano le 
scelte didattiche e disciplinari operate dagli insegnanti di questi livelli scolastici (Canducci et al., 2020). Questa considerazione porta a ipotizzare che alla mancanza di gradualità dei passaggi nei libri di testo italiani da modalità argomentative del far "fare" a modalità argomentative del far "immaginare" (tra la III SE e la IV SE) e da modalità argomentative del far "fare" a modalità argomentative del far "astrarre" (tra la V SE e la I SM) possa corrispondere una mancanza di gradualità degli stessi passaggi nelle dinamiche e nei modi più diffusi di insegnamento-apprendimento interni alle prassi scolastiche. Ora, poiché non è affatto scontato che questi balzi verso l'immaginare e verso l'astrarre si affianchino a corrispondenti balzi cognitivi degli studenti che passano dalla III SE alla IV SE, e dalla V SE alla I SM, ci sembra che questa mancanza di gradualità sia poco giustificabile in termini didattici.

Rispetto al contesto italiano, in cui globalmente i Movimenti logico-argomentativi rappresentano circa un quarto dei Movimenti testuali totali, in Canton Ticino e nel Canton Grigioni emerge come i libri di testo che trattano l'argomento "poligoni" prediligano meno questa tipologia di Movimento testuale, pur essendo importante per accompagnare i ragionamenti degli allievi, e che tali Movimenti sono proposti senza troppi salti cognitivi tra una classe e l'altra; questo è indicatore del fatto che questi libri "parlano" al lettore in modo diverso.

Lo studio qui presentato non vuole dunque solo essere una ricognizione teorico-descrittiva, ma vuole offrire a insegnanti e a ricercatori interrogativi e stimoli da cui partire per sperimentare in classe, acquisendo il gusto di entrare nella proposta testuale con gli allievi, osservandola, smontandola o anche semplicemente esprimendosi su di essa, per cercare di capire quali sono le loro reali necessità e difficoltà in vista di un apprendimento che non sia banale ripetizione (quando c'è), ma vera interiorizzazione.

\section{Bibliografia}

Antonini, S. (2011). Generating examples: focus on processes. ZDM - Mathematics Education, 43, $205-217$. https://doi.org/10.1007/s11858-011-0317-6

Antonini, S., Presmeg, N., Mariotti, M. A., \& Zaslavsky, O. (2011). On examples in mathematical thinking and learning. ZDM - Mathematics Education, 43(2), 191-194. https://doi.org/10.1007/s11858-011-0334-5

Austin, J. L. (1987). Come fare cose con le parole. Marietti Editore. (Titolo originale: How to do things with words pubblicato nel 1962).

Balacheff, N. (1987). Processus de preuve et situations de validation. Educational Studies in Mathematics, 18(2), $147-176$.

Balacheff, N. (1988). Une étude des processus de preuve en mathématiques chez les élèves de Collège. Thèse d'état. Université Joseph Fourier.

Balacheff, N. (2001). Imparare la prova. Pitagora Editrice.

Barsalou, L. W. (2008). Grounded cognition. Annual Review of Psychology, 59, 617-645.

Berruto, G. (1987). Sociolinguistica dell'italiano contemporaneo. La Nuova Italia Scientifica.

Boero, P., Garuti, R., \& Mariotti, M. A. (1996). Some dynamic mental processes underlying producing and proving conjectures. Proceedings of the 20th PME Conference, Valencia, Spain, 2, 121-128.

Buchbinder, O., \& Zaslavsky, O. (2011). Is this a coincidence? The role of examples in fostering a need for proof. ZDM - Mathematics Education, 43(2), 269-281.

Canducci, M. (2019). II rapporto testo-figure nei libri di testo di matematica: il caso dei poligoni analizzato in 
ottica multimodale, In B. D'Amore \& S. Sbaragli (A cura di), Didattica della matematica e professionalità docente, Atti del XXXIII convegno di Castel San Pietro Terme (pp. 107-108). Pitagora.

Canducci, M., Demartini, S., Franchini, E., \& Sbaragli, S. (2019a). La definizione nei testi scolastici: dall'analisi alla didattica. In B. Di Paola (A cura di), Pratiche d'aula e ricerca didattica: nuove e vecchie sfide di insegnamento/ apprendimento matematico per una scuola competente e inclusiva (pp. 47-48). "Quaderni di Ricerca in Didattica", n. 2 Numero speciale n. 5, 2019. G.R.I.M. http://math.unipa.it/grim/quaderno2 suppl 5 2019.pdf

Canducci, M., Demartini, S., Franchini, E., \& Sbaragli, S. (2019b). Analisi di manuali scolastici di matematica dal punto di vista linguistico e disciplinare. In B. Di Paola (A cura di), Pratiche d'aula e ricerca didattica: nuove e vecchie sfide di insegnamento/apprendimento matematico per una scuola competente e inclusiva (pp. 4344). "Quaderni di Ricerca in Didattica", n. 2 Numero speciale n. 5, 2019. G.R.I.M. http://math.unipa.it/ grim/quaderno2 suppl 5 2019.pdf

Canducci, M., Demartini, S., Franchini, E., \& Sbaragli, S. (2020). I materiali didattici che vorrei: il punto di vista dei docenti di matematica. Scuola ticinese, No. 337: Anno XLIX, Serie IV, 1/2020, 57-62.

Canducci, M., Demartini, S., \& Sbaragli, S. (in stampa). Plurale o singolare? Disomogeneità linguistica di numero nei manuali di matematica della scuola primaria e secondaria di primo grado italiani. Italiano a scuola, 3.

Canducci, M., Rocci, A., \& Sbaragli, S. (in stampa). The influence of multimodal textualization in the conversion of semiotic representations in Italian primary school geometry textbooks. Multimodal Communication. De Gruyter.

Cardona, G. R. (2006). Introduzione all'etnolinguistica. UTET.

Cattani, A. (1994). Forme dell'argomentare. I/ ragionamento tra logica e retorica. Edizioni GB.

Colombo, A. (1992). Per una definizione e analisi pragmatica dei testi argomentativi. In G. Gobber (A cura di), La linguistica pragmatica (pp. 475-500). Bulzoni.

Corno, D. (2011). "retorica". In Enciclopedia dell'Italiano Treccani diretta da R. Simone, vol. II. Istituto dell'Enciclopedia. https://www.treccani.it/enciclopedia/retorica (Enciclopedia-dell\%27/taliano)/

Cortelazzo, M. (1994). Testo scientifico e manuali scolastici. In M. L. Zambelli (A cura di), La rete e i nodi. I/ testo scientifico nella scuola di base (pp. 3-14). Quaderni del Giscel. La Nuova Italia.

D’Ambrosio, U. (2002). Etnomatematica. Pitagora.

D’Amore, B., \& Sbaragli, S. (2017). La matematica e la sua storia. Dalle origini al miracolo greco. Dedalo.

D’Amore B., \& Sbaragli, S. (2018). La matematica e la sua storia: dal tramonto greco al medioevo. Dedalo.

D’Amore B., \& Sbaragli, S. (2019). La matematica e la sua storia: dal rinascimento al XVIII secolo. Dedalo.

D’Amore B., \& Sbaragli, S. (2020). La matematica e la sua storia. Dal XVIII al XXI secolo. Dedalo.

Demartini, S., \& Sbaragli, S. (2019). Le parole che "ingannano". La componente lessicale nell'insegnamento e nell'apprendimento della matematica. In B. Di Paola (A cura di), Pratiche d'aula e ricerca didattica: nuove e vecchie sfide di insegnamento/apprendimento matematico per una scuola competente e inclusiva (pp. 1925). "Quaderni di Ricerca in Didattica", n. 2 Numero speciale n. 5, 2019. G.R.I.M. http://math.unipa.it/ grim/quaderno2 suppl 5 2019.pdf

Demartini, S., Sbaragli, S., \& Ferrari, A. (2020). L'architettura del testo scolastico di matematica per la scuola primaria e secondaria di primo grado, Italiano LinguaDue, 12(2), 160-180. https://riviste.unimi.it/index.php/ promoitals/article/view/14979/13889

Demartini, S., Fornara, S., \& Sbaragli, S. (2020). Se la sintesi diventa un problema. Alcune caratteristiche del linguaggio specialistico della matematica in prospettiva didattica. In J. Visconti, M. Manfredini \& L. Coveri (A 
cura di), Linguaggi settoriali e specialistici: sincronia, diacronia, traduzione, variazione (pp. 487-494). Atti del XV Congresso Internazionale SILFI, 28-30 maggio 2018. Cesati.

Dressler, W. U., \& Beaugrande, R. (1984). Introduzione alla linguistica testuale. il Mulino.

Duval, R. (1998). Argomentare, dimostrare, spiegare: continuità o rottura cognitiva? Pitagora.

Ellis, A. B., Ozgur, Z., Vinsonhaler, R., Dogan, M. F., Carolan, T., Lockwood, E., Lynch, A., Sabouri, P., Knuth, E., \& Zaslavsky, O. (2019). Student thinking with examples: The criteria-affordances-purposes-strategies framework. The Journal of Mathematical Behavior, 53, 263-283.

Enriques, F. (1906). Problemi della scienza. Zanichelli.

Ferrari, A. (2014). La linguistica del testo. Principi, fenomeni, strutture. Carocci.

Ferrari, A. (2019). Che cos'è un testo. Carocci.

Ferrari, A., \& Zampese, L. (2016). Grammatica: parole, frasi, testi dell'italiano. Carocci.

Ferrari, P. L. (2004). Matematica e linguaggio. Quadro teorico e idee per la didattica. Pitagora.

Ferreiro, E., \& Teberosky, A. (1985). La costruzione della lingua scritta nel bambino. Giunti Barbera.

Fischbein, E. (1982). Intuition and Proof. For the Learning of Mathematics, 3(2), 9-18.

Garuti, R. (2003). L'Unità cognitiva fra argomentare e dimostrare. L'insegnamento della Matematica e delle Scienze Integrate, 26A(5), 523-540

Godino, A. (2009). L'arte della persuasione: seduzioni del pensiero. Psychofenia: Ricerca ed Analisi Psicologica, 20, 97-128.

Gruppo $\mu$ (1976). Retorica generale. Le figure della comunicazione. Bompiani. (Titolo originale: Réthorique générale pubblicato nel 1970).

Hampe, B. (2017). Metaphor: Embodied Cognition and Discourse. Cambridge University press.

lacona, A. (2005). L'argomentazione. Einaudi.

Kopperschmidt, J. (1985). An Analysis of Argumentation. In T. A. van Dijk (Ed.), Handbook of Discourse Analysis, vol. II, Dimensions of Discourse (pp. 159-168). Academic Press.

Lakoff, G., \& Nùñez, R. (2005). Da dove viene la matematica. Come la mente embodied dà origine alla matematica. Bollati Boringhieri. (Titolo originale: Where Mathematics Comes From: How the Embodied Mind Brings Mathematics into Being pubblicato nel 2000).

Lala, L. (2011). "testo, tipi di". In Enciclopedia dell'Italiano Treccani diretta da R. Simone, vol. II. Istituto dell'Enciclopedia. https://www.treccani.it/enciclopedia/tipi-di-testo (Enciclopedia-dell\%27/taliano)/

Lausberg, H. (1969). Elementi di retorica. il Mulino. (Titolo originale: Elemente der Literarischen Rhetorik pubblicato nel 1949).

Lawson A. E., \& Renner, J. W. (1975). Relationships of concrete and formal operational science subject matter and the developmental level of the learner, Journal of research in science teaching, 12, 347-358.

Lo Cascio, V. (1991). Grammatica dell'argomentare. Strategie e strutture. La Nuova Italia.

Lumbelli, L. (2009). La comprensione come problema. Il punto di vista cognitivo. Laterza.

Marazzini, C. (2001). Il perfetto parlare. La retorica in Italia da Dante a Internet. Carocci. 
Mariotti, M. A. (2006). Proof and proving in mathematics education. In A. Gutiérrez \& P. Boero (Eds.), Handbook of research on the psychology of mathematics education: Past, present and future (pp. 173-204). Sense Publishers.

Martinez, M. V., \& Pedemonte, B. (2014). Relationship between inductive arithmetic argumentation and deductive algebraic proof. Educational Studies in Mathematics, 86(1), 125-149.

Mejía-Ramos, J. P., \& Weber, K. (2020). Using task-based interviews to generate hypotheses about mathematical practice: mathematics education research on mathematicians' use of examples in proof-related activities. ZDM - Mathematics Education, 52, 1099-1112.

Miyazaki, M., Fujita, T., \& Jones, K. (2017). Students' understanding of the structure of deductive proof. Educational Studies in Mathematics, 94(2), 223-239.

Mortara Garavelli, B. (1988). Textsorten/Tipologia di testi. In G. Holtus, M. Metzeltin \& C. Schmitt (Eds.), Lexikon der Romanistischen Linguistik (pp. 157-168). Niemeyer.

Mortara Garavelli, B. (2003). Manuale di retorica. Bompiani. (Edizione originale pubblicata nel 1988).

Pedemonte, B. (2008). Argumentation and algebraic proof. ZDM - Mathematics Education, 40(3), 385-400.

Pedemonte, B., \& Buchbinder, O. (2011). Examining the role of examples in proving processes through a cognitive lens: the case of triangular numbers. ZDM - Mathematics Education, 43(2), 257-267.

Perelman, C., \& Olbrechts-Tyteca, L. (2013). Trattato dell'argomentazione. La nuova retorica. Giulio Einaudi editore. (Titolo originale: La nouvelle rhétorique. Traité de l'Argumentation pubblicato nel 1958).

Piaget, J. (1972). Intellectual evolution from adolescence to adulthood. Human Development, 15, 1-12.

Rigotti, E., \& Greco, S. (2009). Argumentation as an Object of Interest and as a Social and Cultural Resource. In N. M. Mirza \& A.-N. Perret-Clermont (Eds.), Argumentation and Education: Theoretical Foundations and Practices (pp. 1-61). Springer.

Robutti, O. (2006). Embodied cognition e didattica della matematica. La matematica e la sua didattica, 2, $163-186$.

Rousseau, J. J. (1950). Emilio e altri scritti pedagogici. Sansoni. (Titolo originale: Emile ou de l'éducation pubblicato nel 1898).

Sbaragli, S., Demartini, S., Franchini, E., \& Canducci, M. (2020). Grado di soddisfazione e utilizzo del libro di testo di matematica da parte dei docenti di scuola primaria italiana. Form@re - Open Journal per la formazione in rete, 20(3), 132-153. http://dx.doi.org/10.13128/form-9244

Sbisà, M. (1989). Linguaggio, ragione, interazione. il Mulino.

Serianni, L. (2003). Italiani scritti. il Mulino.

Serianni, L. (2010). "lingua scritta". In Enciclopedia dell'Italiano Treccani diretta da R. Simone, vol. I. Istituto dell'Enciclopedia. https://www.treccani.it/enciclopedia/lingua-scritta (Enciclopedia-dell\%27/taliano)/

Toulmin, S. E. (1975). Gli usi dell'argomentazione. Rosenberg \& Sellier. (Titolo originale: The uses of argument pubblicato nel 1958).

Van Hiele, P. M. (1986). Structure and insight. A theory of Mathematics Education. Academic Press.

Watson, A., \& Mason, J. (2006). Mathematics as a constructive activity: Learners generating examples. Routledge.

Werlich, E. (1982). A text grammar of English. Quelle \& Meyer. 